\title{
Locomotion approach of bipedal robot utilizing passive wheel without swing leg based on stability margin maximization and fall prevention functions
}

\author{
Kohei Kimura $^{1 *} \mathbb{0}$, Noriaki Imaoka², Shintaro Noda ${ }^{1}$, Yohei Kakiuchi ${ }^{1}, K^{2}$ O Okada ${ }^{1}$ and Masayuki Inaba ${ }^{1}$
}

\begin{abstract}
This paper proposes a locomotion approach of leg-wheel robot utilizing passive wheel attached to the foot of bipedal robot. The key feature of this approach is bipedal mobility without swing leg. This mobility contributes the stability based on expansion of support polygon during locomotion, the robustness for obstacles and stopping to prevent fall, and the adaptability by prevention of body swing sideways. To achieve these, we propose the stability margin maximization to optimize center of gravity projection for support polygon and the fall prevention functions for real environment that is a difficult situation to prevent unexpected fall by the only planning. Finally, we apply the proposed methods to leg-wheel phases through locomotion and verify the contribution by experiments using real bipedal robot.
\end{abstract}

Keywords: Locomotion, Leg-wheel robot, Stability margin maximization, Fall prevention functions

\section{Introduction}

There are some studies of leg-wheel robots that are composed of the integration of legged robots and wheeled robots [1-3]. Legged robots have the advantage of versatile gait locomotion for various environments and wheeled robots have the advantage of stable and efficient locomotion. As the previous researches related to leg-wheel robots, the quadruped type [1] and the biped type $[2,3]$ are proposed. The biped type leg-wheel robots enable the transformation between bipedal walking and wheel locomotion by utilizing the stewart platform mechanisms of legs [2] and having the additional actuators in knees as active driven wheels [3]. In this paper, we aim the establishment of the locomotion approach of legwheel robot that slides the foot by rolling passive wheel

\footnotetext{
*Correspondence: k-kimura@jsk.imi.i.u-tokyo.ac.jp

1 Department of Mechano-Informatics, Graduate School of Information Science and Technology, The University of Tokyo, 7-3-1, Hongo,

Bunkyo-ku, Tokyo 113-8656, Japan

Full list of author information is available at the end of the article
}

attached to the heel. Without the complex linkage mechanisms and the additional actuators, this locomotion approach is simply realized by only attaching the passive wheel to the heel of bipedal robot as shown in Fig. 1. There are several merits to utilize passive wheel for legwheel robot as well as the simplicity of attachment. Even if the general leg-wheel robot is unable to drive its wheels actively due to the accident of wheel drive system and the road surface conditions, the leg-wheel robot equipped with passive wheel has the possibility of locomotion by driving other joints of legs. Furthermore, integrating passive wheel into robot system enables the compliance locomotion for external environments because passive wheel joints relieve the internal force applied inside the closed link system consisting of legs and ground.

Having no swing leg is the key feature of the proposed locomotion approach of bipedal robot utilizing passive wheel. This feature contributes (1) expansion of support polygon during locomotion, (2) robustness and stopping for obstacles, and (3) prevention of body swing sideways. 


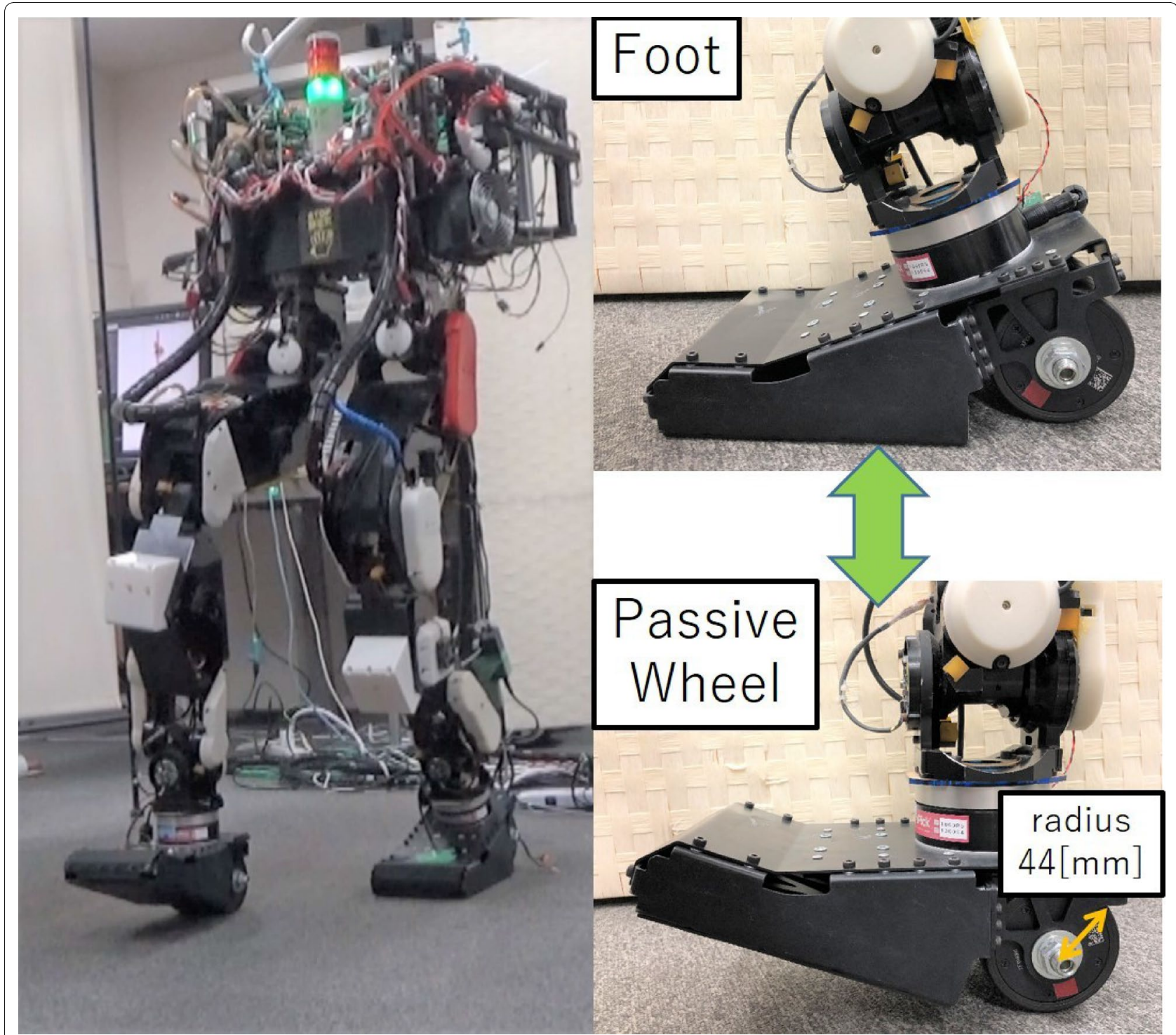

Fig. 1 Locomotion approach of bipedal robot utilizing passive wheel without swing leg

Section "Key features of locomotion without swing leg by utilizing passive wheel" describes these features in detail. Section "Stability margin maximization" proposes the planning to optimize center of gravity projection of robot to achieve the locomotion. Section "Fall prevention functions" proposes the strategies to prevent fall in the real environment that is a difficult situation to prevent by the only planning. Section "Experiments" shows the verification and feasibility of the proposed locomotion approach by using real bipedal robot.

\section{Key features of locomotion without swing leg by utilizing passive wheel}

By virtue of utilizing passive wheel and having no swing leg, a bipedal robot benefits from the features of locomotion as shown in Fig. 2.

\section{Expansion of support polygon during locomotion}

The locomotion rolling passive wheel on the ground expands support polygon of robot because of no swing leg as shown in Fig. 2-I. Figure 2-I is the bottom view of bipedal robot and the support polygon is generated by applying convex hull to the contact region of robot and ground. To maintain support polygon extensively implies 


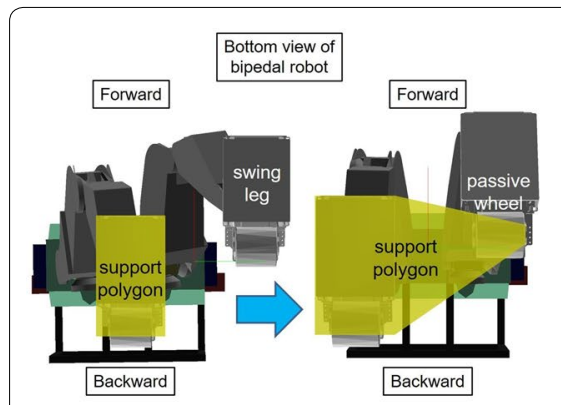

(I) Expansion of support polygon

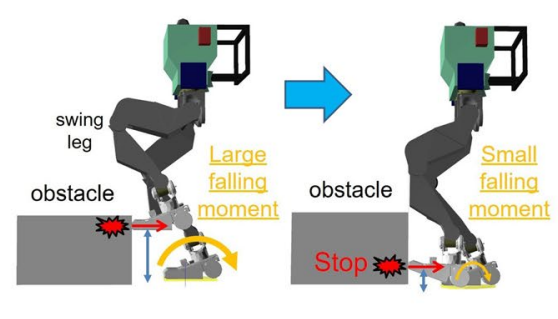

(II) Robustness and stopping

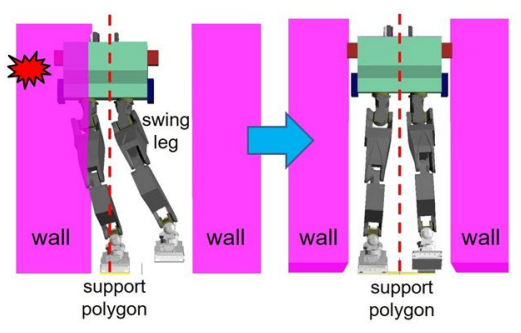

(III) Prevention of body swing sideways

Fig. 2 Key features and contribution of locomotion without swing leg

the large tolerance that center of gravity projection point, Zero Moment Point (ZMP) [4], and Capture Point [5] can exist. Capture Point is utilized as an indicator not to fall. To take these points into consideration is necessary to keep balance of robot and contributes the stable locomotion.

\section{Robustness and stopping for obstacles}

As shown in Fig. 2-II, we compare the case of having swing leg with the case of having no swing leg in the situation where robot collides with obstacle during locomotion. In the case that swing leg of robot collides with obstacle in the left of Fig. 2-II, the external force by impact is applied above the level of support region and robot tends to fall due to the large overturning moment around support region. By contrast, in the case of locomotion without swing leg by sliding passive wheel in the right of Fig. 2-II, the point of external force applied by impact can be always kept in the lower position. Therefore, even if the external force on the same level with the former case is applied, the overturning moment around support region is reduced smaller and robot acquires the robustness.

In the case of dynamic walking in the left of Fig. 2-II, robot is unable to stop immediately after the collision with obstacle is detected, and the complex processing for fall avoidance such as foot step modification [6] is required. On the contrary, the static locomotion approach in the right of Fig. 2-II has the feature that robot is able to stop with holding its pose immediately after the collision with obstacle occurs. As other features, to put swing leg always on the ground contributes the avoidance from the accident that robot steps on obstacle and falls over.

\section{Prevention of body swing sideways}

The walking of general bipedal robot has the problem that the body of robot swings sideways. This is caused by the sideways trajectory planning and generation of center of gravity projection and ZMP so that these points are included in support polygon as shown in the left of Fig. 2-I while the support polygon for right foot and left foot switches alternately. Fig. 2-III shows the situation that bipedal robot passes through narrow space between walls. As shown in the left of Fig. 2-III, the normal bipedal walking causes the body swing sideways and consequently, the body links of robot interfere with walls. In contrast, the center of gravity projection onto the expanded support polygon in the right of Fig. 2-I enables robot to prevent the lateral swing of body and achieve the passing through narrow space as shown in the right of Fig. 2-III.

Besides the above effectiveness, the prevention of body swing sideways contributes the fall prevention of the load mounted on robot and the prevention of the horizontal blur of robot vision.

Utilizing the slipperiness of passive wheel reduces the high friction and the high load such as the shuffle motion [7] of bipedal robot and enables the smaller shaking locomotion than the shuffle motion requiring the complex distribution of sole contact force and frictional force.

\section{Stability margin maximization}

In this section, we describe the optimization planning how to project the center of gravity of bipedal robot onto the support polygon expanded by utilizing passive wheel.

\section{Definition}

In general, when a center of gravity projection point of robot exists in a support polygon, robot is statically stable. Here, the center of gravity projection point is a foot of a perpendicular from the center of gravity of robot to the ground. Accordingly, when the center of gravity of 
robot is projected onto a certain point located within a support polygon, the planning that stability is guaranteed can be implemented. We consider the optimal position of a certain point located within a support polygon. This optimal position is the target of center of gravity projection. The indicator to optimize in this paper is the stability and the idea of stability margin [8] is applied as stability criterion and quantitative evaluation. Therefore, we define stability margin maximization to maximize the stability margin as objective function.

Stability margin is represented as the distance between center of gravity projection point and boundary line of support polygon. Namely, stability margin $S(x, y)$ equals the minimum value among the distance $d_{i}(x, y)$ between center of gravity projection point $(x, y)$ and each boundary line $i$ of support polygon in the following formula.

$$
S(x, y)=\min _{i} d_{i}(x, y)
$$

For the static state, the center of gravity projection point $(x, y)$ is in the set $P$ composed of all points within support polygon. Let stability margin maximum point $\boldsymbol{p}_{\max }$ be the optimum point where stability margin $S(x, y)$ is maximum, and $\boldsymbol{p}_{\max }$ is defined as follows.

$$
\boldsymbol{p}_{\text {max }}=\underset{(x, y) \in P}{\arg \max } S(x, y)=\underset{(x, y) \in P}{\arg \max }\left\{\min _{i} d_{i}(x, y)\right\}
$$

Stability margin maximization is to provide the stability margin maximized by solving $\boldsymbol{p}_{\max }$ in Eq. (2).

\section{Solution}

To apply stability margin maximization to real robot, we need to solve the problem Eq. (2). When a support polygon has an inscribed circle, $\boldsymbol{p}_{\max }$ in Eq. (2) geometrically corresponds to the center of this inscribed circle. However, the case that the support polygon of real robot does not have an inscribed circle exists. Therefore, we formalize Eq. (2) into the generalized algorithm that is possible to be solved by computer. We generalize the support polygon of robot as shown in Fig. 2 -I and show $n$-sided support polygon in Fig. 3 . Let $i$ be $i=0,1, \ldots, n-1$.

Equation (2) can be interpreted as the general optimization problem composed of objective function and constraint conditions. First, we assume that stability margin maximization is achieved with respect to a certain boundary line $i$. Let $\boldsymbol{p}_{\max }^{i}$ be the stability margin maximum point for $i$. $\boldsymbol{p}_{\max }^{i}$ is obtained as 2 variables $x, y$ to maximize $d_{i}(x, y)$ (Objective Function). Meanwhile, from the definition Eq. (1) of stability margin, $d_{i}(x, y)$ should be smaller than any other distance $d_{j}(x, y), \forall j \notin i$ (Constraint Condition 1). Moreover, the inclusive condition $(x, y) \in P$ for support polygon should be satisfied, which is provided as the precondition in Eq. (2). Based on Fig. 3, this condition

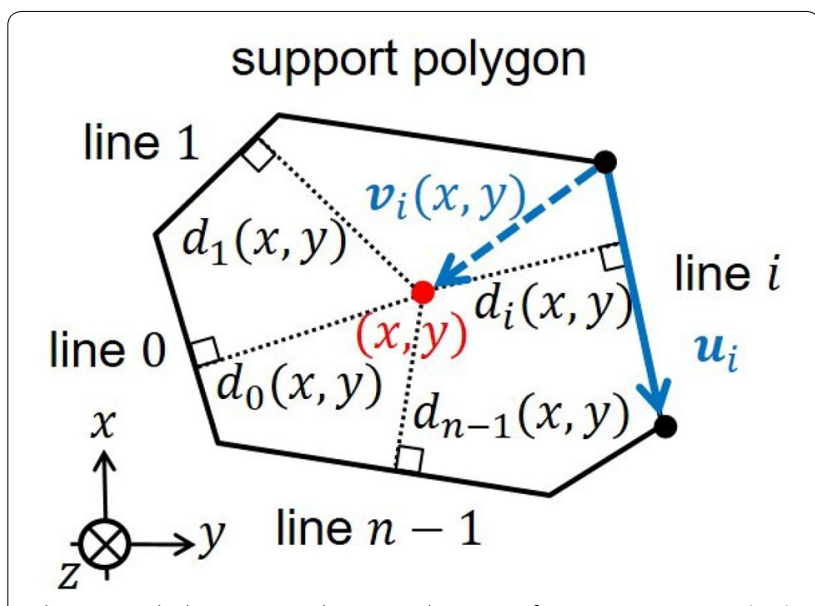

Fig. $3 n$-sided support polygon and center of gravity projection $(x, y)$

is equivalent to the condition that $\boldsymbol{u}_{k} \times \boldsymbol{v}_{k}(x, y) \geq 0$ is satisfied for all boundary lines $\forall k=0,1, \ldots, i, \ldots, n-1$ (Constraint Condition 2). Here, $\boldsymbol{u}_{k}$ represents the vector from the start point to the end point of the boundary line $k$ when turning clockwise round the boundary lines of support polygon, and $\boldsymbol{v}_{k}(x, y)$ represents the vector from the start point of the boundary line $k$ to an arbitrary point $(x, y)$. Each component of the vectors follows the coordinate system in Fig. 3.

From the above, the optimization problem with respect to $\boldsymbol{p}_{\max }^{i}$ is as follows.

$$
\begin{aligned}
\boldsymbol{p}_{\max }^{i}= & \underset{x, y}{\arg \max } d_{i}(x, y) \\
\text { s.t. } & \forall j \notin i, \quad d_{i}(x, y) \leq d_{j}(x, y) \\
& \forall k, \quad \boldsymbol{u}_{k} \times \boldsymbol{v}_{k}(x, y) \geq 0
\end{aligned}
$$

$\boldsymbol{p}_{\max }^{i}$ represents the center of the largest circle that contacts with the boundary line $i$ and is included in the support polygon. $d_{i}(x, y)$ is denoted by $\boldsymbol{u}_{i}$ and $\boldsymbol{v}_{i}(x, y)$ from the geometric relationship in Fig. 3 and its absolute value can be removed from the Constraint Condition 2 in Eq. (3). Besides, note that $\boldsymbol{u}_{i} \times \boldsymbol{v}_{i}(x, y)$ is the linear expression for $x, y . d_{i}(x, y)$ is as follows.

$$
d_{i}(x, y)=\frac{\left|\boldsymbol{u}_{i} \times \boldsymbol{v}_{i}(x, y)\right|}{\left|\boldsymbol{u}_{i}\right|}=\frac{\boldsymbol{u}_{i} \times \boldsymbol{v}_{i}(x, y)}{\left|\boldsymbol{u}_{i}\right|}=\frac{a_{i} x+b_{i} y+c_{i}}{\left|\boldsymbol{u}_{i}\right|}
$$

$a_{i}, b_{i}$, and $c_{i}$ are the constants particular to support polygon. By substituting Eq. (4) for Eq. (3), the optimization problem with respect to $\boldsymbol{p}_{\max }^{i}$ is eventually summarized as follows. 


$$
\begin{aligned}
\boldsymbol{p}_{\max }^{i}= & \underset{x, y}{\arg \max } \frac{a_{i} x+b_{i} y+c_{i}}{\left|\boldsymbol{u}_{i}\right|} \\
\text { s.t. } & \forall j \notin i, \quad \frac{a_{i} x+b_{i} y+c_{i}}{\left|\boldsymbol{u}_{i}\right|} \leq \frac{a_{j} x+b_{j} y+c_{j}}{\left|\boldsymbol{u}_{j}\right|} \\
& \forall k, a_{k} x+b_{k} y+c_{k} \geq 0
\end{aligned}
$$

In the optimization problem Eq. (5), both objective function and constraint conditions are represented as the linear expression for 2 variables $x, y$, and therefore this problem can be solved by Linear Programming (LP). In this paper, we utilize qpOASES [9] as the LP solver library.

Finally, we obtain $\boldsymbol{p}_{\max }$ from $\boldsymbol{p}_{\max }^{i}$ as follows.

$$
\boldsymbol{p}_{\max }=\boldsymbol{p}_{\max }^{i_{\max }}, \quad i_{\max }=\underset{i}{\arg \max } d_{i}\left(\boldsymbol{p}_{\max }^{i}\right)
$$

$d_{i}\left(\boldsymbol{p}_{\max }^{i}\right)$ represents the distance between $\boldsymbol{p}_{\max }^{i}$ and the boundary line $i$ and geometrically implies the radius of the largest circle that contacts with the boundary line $i$ and is included in the support polygon.

\section{Application to leg-wheel phases}

We practically apply stability margin maximization to bipedal robot with passive wheel. We clarify the definition of support polygon for this leg-wheel robot.
As shown in Fig. 4, two types of support polygons are assumed for this robot. The support polygon $P$ is generated by applying convex hull to the contact region including wheels and the support polygon $Q$ is composed of the only feet soles. We compare $P$ with $Q$ in terms of the stability when the center of gravity $(x, y)$ of robot moves posterior to the stance foot. In Fig. 4, four poses (1) (4) of robot are generated by inverse kinematics for the center of gravity $(x, y)$. The relationship between $(x, y)$ and $P, Q$ is (1): $(x, y) \in P,(x, y) \in Q$, (2): $(x, y) \in P,(x, y) \in \operatorname{bd}(\mathrm{Q})$, (3): $(x, y) \in P,(x, y) \notin Q$, and (4): $(x, y) \in \operatorname{bd}(\mathrm{P}),(\mathrm{x}, \mathrm{y}) \notin \mathrm{Q}$, respectively. Here, $\mathrm{bd}(*)$ represents the boundary of set $*$. For the poses (1) (3), the robot can keep the posture on the slippery tile floor, especially the robot has not fallen in (3) even though the center of gravity of robot locates posterior to the edge of the feet soles $((x, y) \notin Q)$. For the pose (4), the robot falls backward because the center of gravity locates in the boundary of $P((x, y) \in \operatorname{bd}(\mathrm{P}))$. Therefore, the support polygon that the center of gravity of robot can exist without falling is defined as $P$ in this paper.

We define the phase that one leg of bipedal robot lands a sole and the other leg lands a passive wheel as leg-wheel phase. As opposed to the phase that both legs land soles,

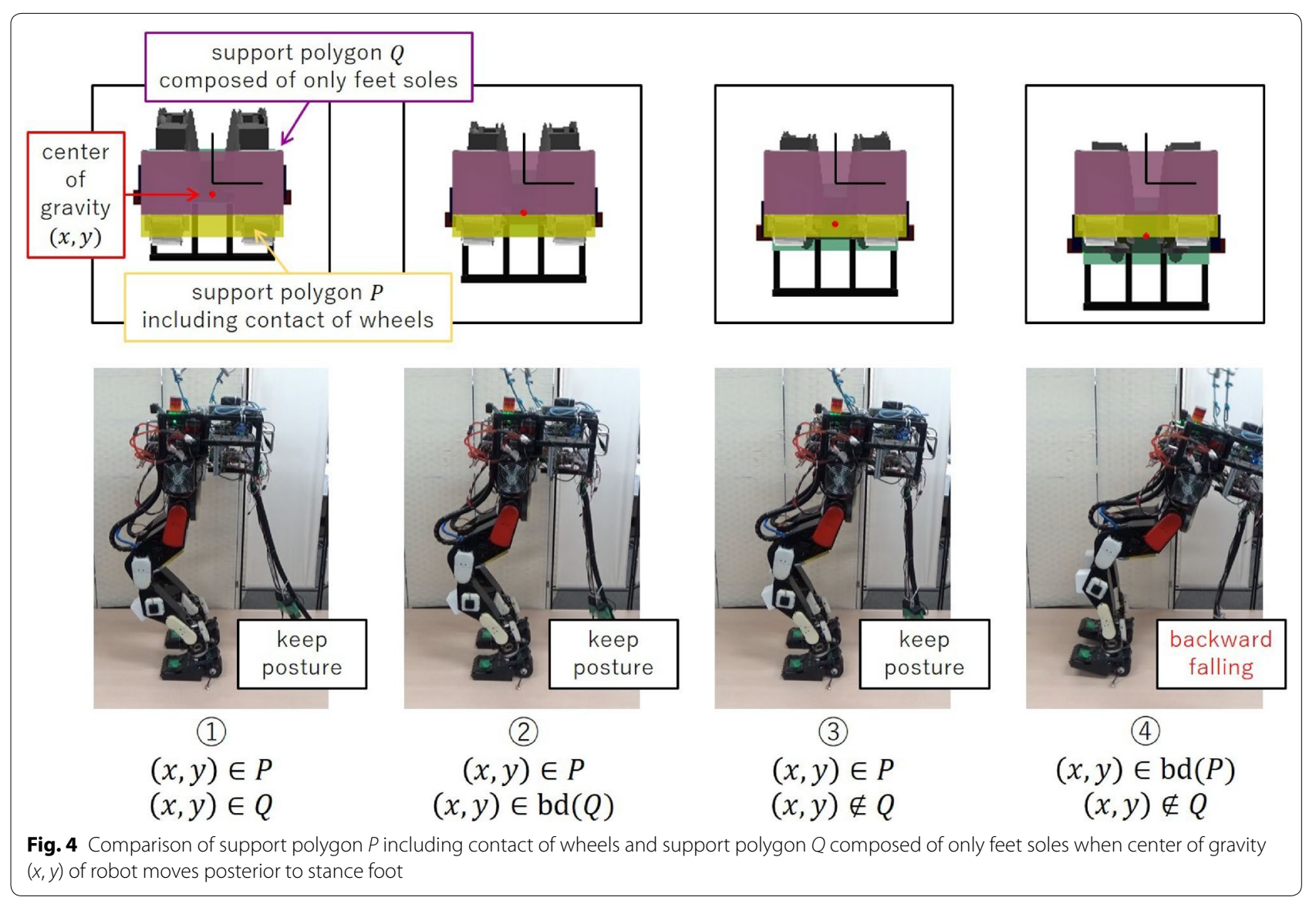




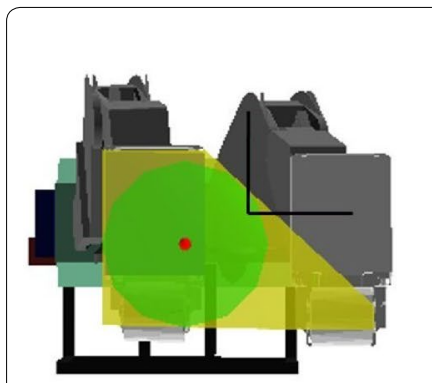

a1 Phase1

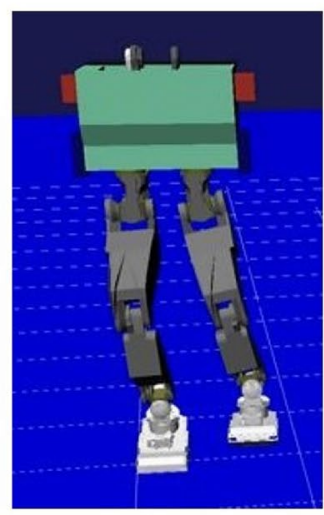

b1Phase1

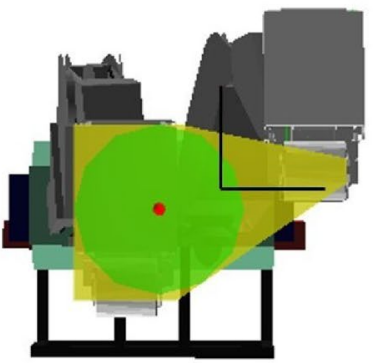

a2 Phase2

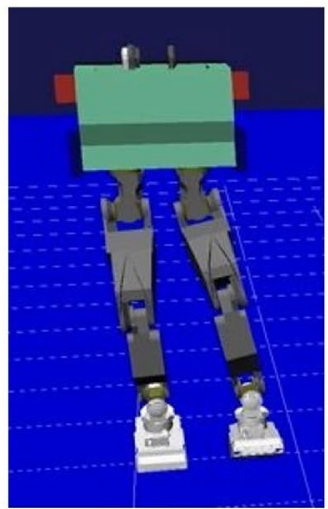

b2 Phase2

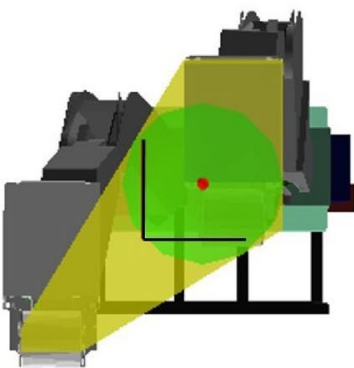

a3 Phase 3

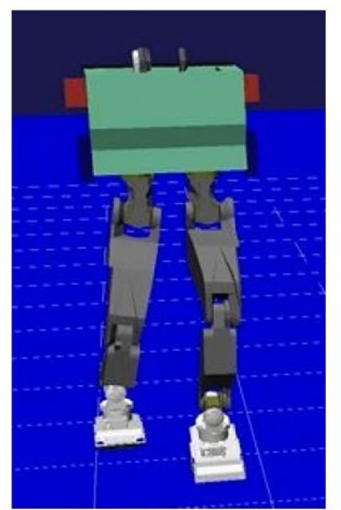

b3 Phase3

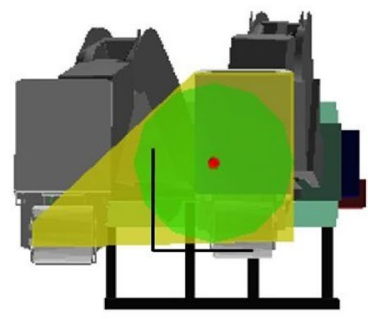

a4 Phase4

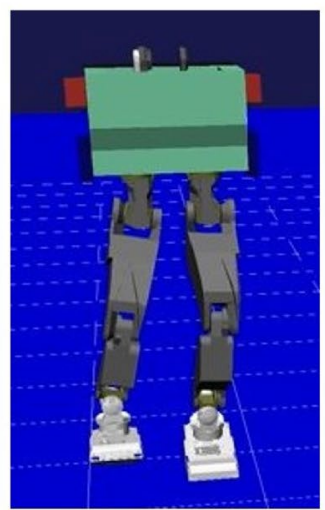

b4 Phase4

Fig. 5 Results of stability margin maximization (a1-a4) and dynamics simulation (b1-b4)

the effectiveness of stability margin maximization for legwheel phase is remarkable because of the difference of right and left contact area. We show the results that stability margin maximization is applied to each leg-wheel phase in Fig. 5. The leg that lands a passive wheel in legwheel phase lifts up the foot around pitch by $20^{\circ}$ keeping the height of the passive wheel axle as shown in the bottom right of Fig. 1. Keeping the height of axle constant guarantees the contact between wheel and ground during swing movement not to float and press the wheel against the ground. By applying stability margin maximization to the support polygons formed from sole and passive wheel on the ground, the stability margin maximum points are obtained as shown in Fig. 5a1-a4. Here, in Fig. 5a1-a4, the black coordinate systems are the world coordinate systems referenced in Fig. 3, the yellow regions are the support polygons, the red points are the stability margin maximum points, and the green circles are the stability margin circles whose radius equals stability margin $S(x, y)$. The stability margin circles in Fig. 5a1-a4 are the circles that have the maximized stability margin. It is observed that the stability margin circles maximized by stability margin maximization represent the largest circles that are included in the support polygons and these circles contact with the several boundary lines of the support polygons. When these circles contact with all boundary lines of the support polygons, these circles correspond to inscribed circles.

By solving inverse kinematics for the center of gravity of robot to be projected onto the obtained stability margin maximum points, the reference joint angles of robot are determined. We show the results of dynamics simulation when these reference joint angles are commanded to bipedal robot on the simulator Choreonoid [10] in Fig. 5b1-b4. It is observed that robots keep balance without falling in all phases. Furthermore, following the sequential commands from Phase1 to Phase2 and from Phase 3 to Phase4, robots are able to slide their passive wheels keeping balance. The swing leg trajectory that rolls passive wheel is determined as simple straight line trajectory because the height of passive wheel axle is kept constant during the sliding of foot. To guarantee the smoothness of the trajectory for the center of gravity of robot through the time, minimumjerk model [11] is adopted as the trajectory algorithm. The minimum-jerk model enables the motion trajectory 


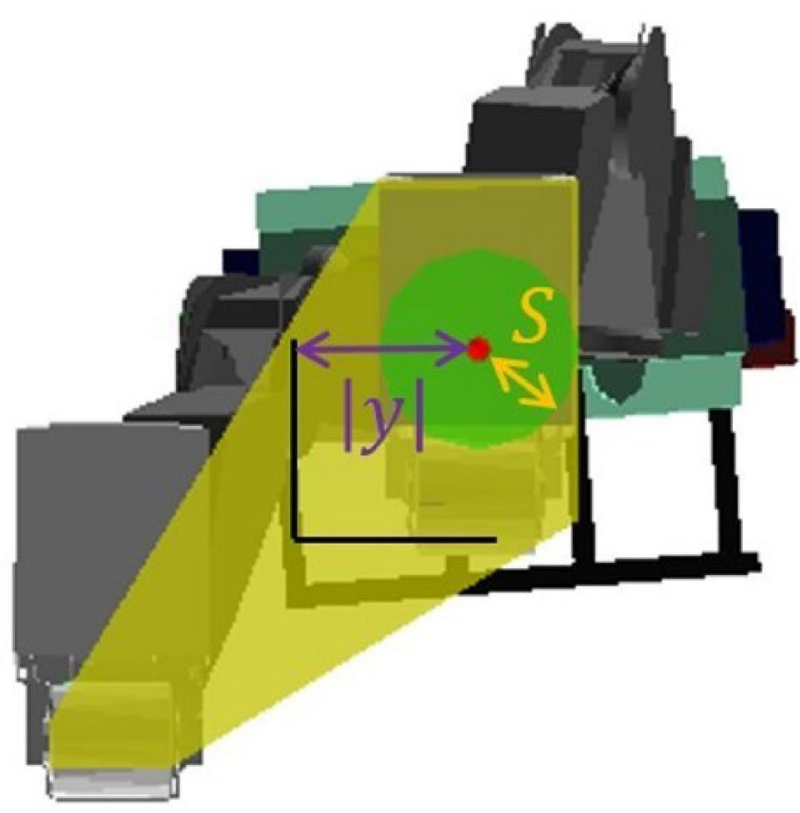

a Definitions of $S$ and $|y|$

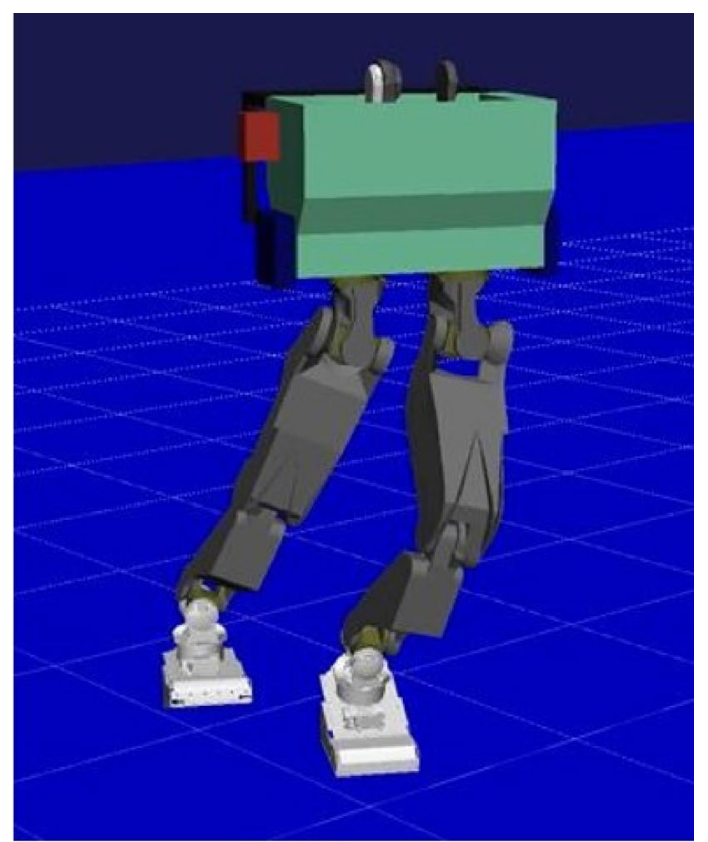

b Simulation

Fig. 6 Single leg stance on Phase3

Table 1 Stability margin $S$, lateral amplitude $|y|$, and force distribution $F_{z}^{R}: F_{z}^{L}$

\begin{tabular}{|c|c|c|c|}
\hline Method & $S(\mathrm{~mm})$ & $|y|(m m)$ & $F_{z}^{\mathrm{R}}: F_{z}^{\mathrm{L}}(\mathrm{N})$ \\
\hline Single leg stance (Fig. 6a) & 76.2 & 139.8 & $0.0: 626.5$ \\
\hline $\begin{array}{l}\text { Stability margin maximization } \\
\text { (Fig. 5a3) }\end{array}$ & 123.4 & 92.5 & 117.3:509.2 \\
\hline With lateral constraint (Fig. 7b) & 104.0 & 35.0 & $241.6: 384.9$ \\
\hline
\end{tabular}

of robot to be interpolated smoothly without loss of balance for sudden change of stability margin maximum point $\boldsymbol{p}_{\max }$ due to the change of support polygon.

Figure 6 represents the single leg stance on Phase3 where the center of gravity of robot is projected onto the center of sole without stability margin maximization. Fig. 6a shows the center of gravity projection point (the red point) and the stability margin circle (the green circle). Considering the radius of stability margin circle (the stability margin) $S$, the lateral amplitude $|y|$, and the normal force distribution applied to right foot and left foot $F_{z}^{\mathrm{R}}: F_{z}^{\mathrm{L}}$, we make a comparison with the result of Fig. $5 \mathrm{a} 3$. We show the comparison result in Table 1. The larger the number of $S$ is, the higher the stability is. This effectiveness is observed in "Stability margin maximization (Fig. 5a3)" in Table 1. The smaller the number of $|y|$ is, the more effective the prevention of body swing sideways is. Similarly, this prevention is observed in "Stability margin maximization (Fig. 5a3)" in Table 1 . This result regarding the lateral amplitude is also observed from the difference of simulation results between Fig. 5b3 and Fig. 6b. Finally, from the result of $F_{z}^{\mathrm{R}}: F_{z}^{\mathrm{L}}$, it is observed that stability margin maximization distributes the robot's own weight to the foot whose wheel is grounded.

\section{Stability margin maximization with lateral constraint}

Although stability margin maximization enables the prevention of body swing sideways, the further prevention is required so that robot can pass through narrower space. In this paper, we propose stability margin maximization with lateral constraint by adding the constraint with respect to $y$ to the constraint conditions of stability margin maximization. This strategy is feasible based on the addition of the following conditional expression to the constraint conditions in Eq. (5).

$$
-\varepsilon \leq y \leq \varepsilon
$$

$\varepsilon$ represents the parameter regarding lateral tolerance limit.

For example Phase3 of leg-wheel phases, we show the comparison result between (A) Without lateral 


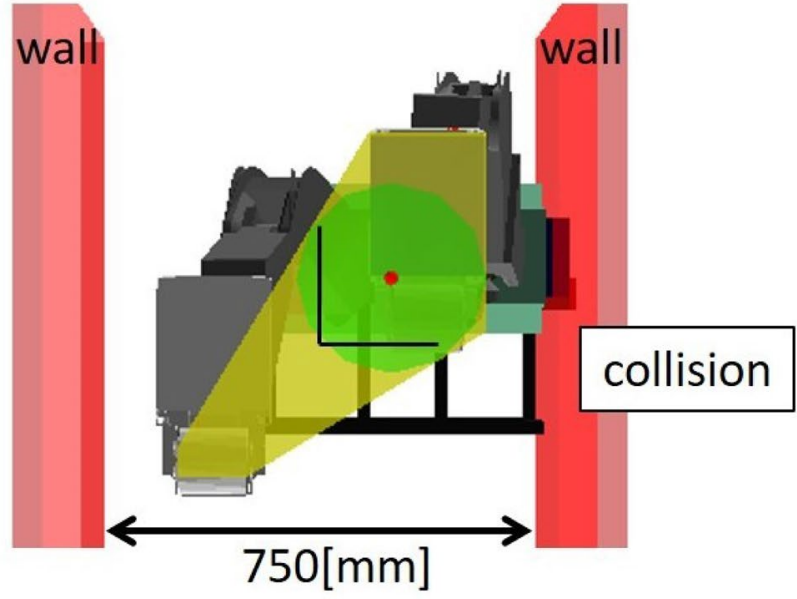

\section{a Without lateral constraint}
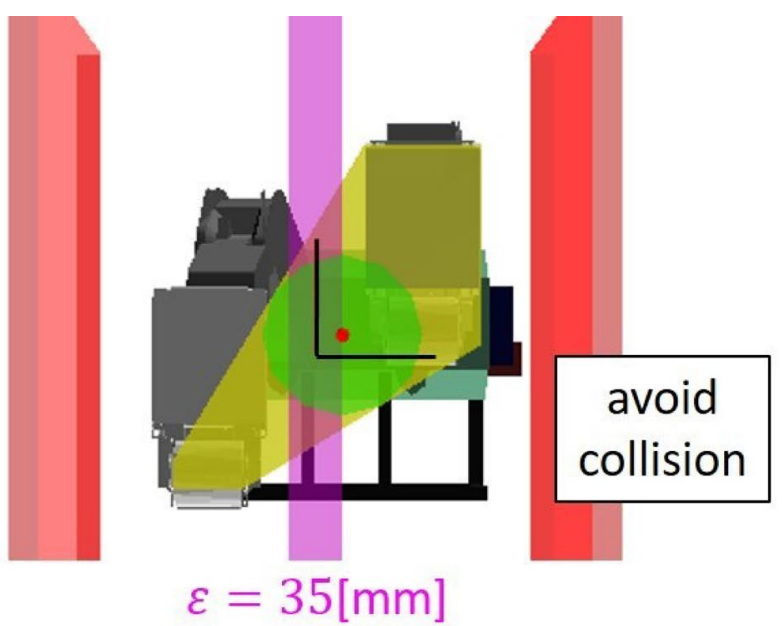

b With lateral constraint

Fig. 7 Comparison at narrow space on Phase3

constraint and (B) With lateral constraint Eq. (7) in Fig. 7. In Fig. 7, the walls of narrow space are highlighted in red and we set the distance between walls as $750 \mathrm{~mm}$. When bipedal robot passes through this space, normal stability margin maximization causes the collision between robot links and wall as shown in Fig. 7a. By contrast, stability margin maximization with lateral constraint of $\varepsilon=35 \mathrm{~mm}$ enables robot to pass through this space without collision based on the smaller swing sideways than (A). We show the result of $S,|y|$, and $F_{z}^{\mathrm{R}}: F_{z}^{\mathrm{L}}$ for Fig. $7 \mathrm{~b}$ in Table 1 . In comparison with the result for normal stability margin maximization (Fig. 5a3), the stability margin $S$ decreases, whereas the lateral amplitude $|y|$ gets smaller and is given as the value of $\varepsilon$. From this observation, it is found out that stability margin maximization with lateral constraint is the trade-off strategy that prevents the body swing sideways more effectively by reducing the stability margin. From the result of $F_{z}^{\mathrm{R}}: F_{z}^{\mathrm{L}}$, it is also observed that the ratio of weight load distribution to the foot whose wheel is grounded increases besides the prevention of body swing sideways.

\section{Fall prevention functions}

In this section, we describe the online-based prevention functions against the fall factors that are difficult to be prevented by only offline planned stability margin maximization.

\section{Emergency Stopper}

Because bipedal robots such as humanoid robots tend to lose balance and fall, the methods to prevent the fall accident are proposed [12-14]. Emergency Stop [12] is one of those methods. Emergency Stop is utilized for fall detection and motion reverting not to fall based on the measurement of Capture Point (CP) [5] $\xi_{x, y}^{\mathrm{CP}}$ defined as the following formula.

$$
\xi_{x, y}^{\mathrm{CP}}=\xi_{x, y}^{\mathrm{COG}}+\dot{\xi}_{x, y}^{\mathrm{COG}} \sqrt{\frac{\xi_{z}^{\mathrm{COG}}-\xi_{z}^{\mathrm{ZMP}}}{g}}
$$

$\xi_{x, y}^{\mathrm{A}}$ represents the vector of $(x, y)$ elements of point $\mathrm{A}$ and $\xi_{z}^{A}$ represents the value of $z$ element of point A. Regarding the point A, CP represents Capture Point, COG represents center of gravity of robot, and ZMP represents

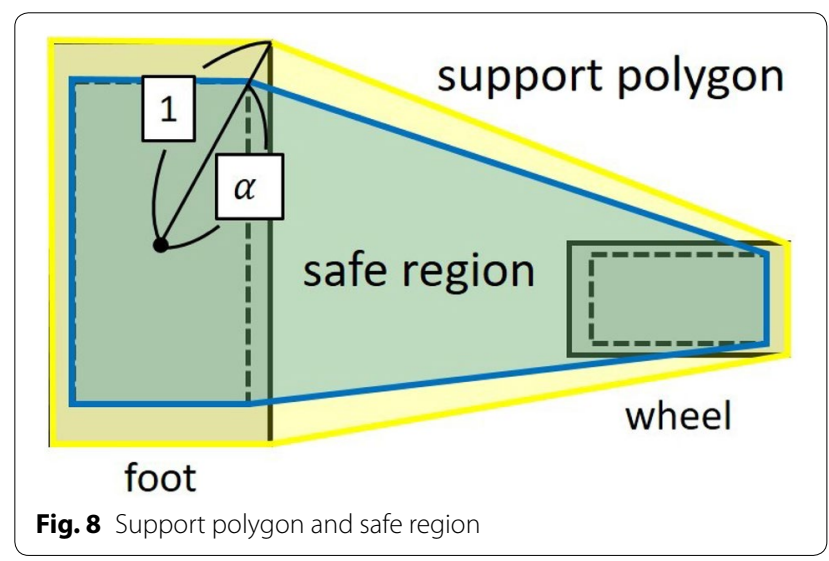


Zero Moment Point, respectively. $g$ represents gravitational acceleration.

In this paper, we define the region with margin from support polygon as Safe Region as shown in Fig. 8 and fall detection works in the moment CP gets out of the Safe Region. And we define the function to stop active motion immediately after fall detection is detected as Emergency Stopper. Safe Region in Fig. 8 is obtained by applying convex hull to the region reduced scale $\alpha(0<\alpha \leq 1)$ for the grounded region of foot and wheel.

\section{Fall prevention function for stumbling}

We describe the problem when bipedal robot stumbles over obstacle during locomotion and propose the strategy to solve this problem.

\section{Problem}

Fig. 9a shows the snapshot when the right foot of real bipedal robot stumbles over the concrete block on the road during the locomotion from Phase 3 to Phase4 in Fig. 5. The real robot falls immediately after this stumbling. Figure 9b shows the state of $\mathrm{CP}$ and Safe Region at this time. The real state of robot measured from joints encoder and attitude sensor is reflected in Fig. $9 \mathrm{~b}$ and we set $\alpha=0.9$. While the real robot falls, it is observed that $\mathrm{CP}$ can not break out of the Safe Region and Emergency Stopper does not work. According to Eq. (8), it is inferred that the impact of stumbling is not transmitted to the change of center of gravity.

\section{Force translation of Safe Region}

As described in Problem, because Emergency Stopper is valid only when CP breaks out of Safe Region, the novel strategy is required for the prevention of fall due to stumbling. Therefore, besed on the inference in Problem, we assume that the virtual force $f$ is applied to center of gravity, which $f$ is the stumbling force applied to foot of robot and can be measured by attached force sensor. And we propose force translation of Safe Region based on the virtual change of center of gravity as shown in Fig. 10. Figure 10 represents the model that robot is approximated by inverted pendulum. Force translation of Safe Region is the technique to translate Safe Region by the change of center of gravity $\Delta x_{\mathrm{SR}}$ that can keep the balance when the horizontal virtual force $f$ is applied to the center of mass

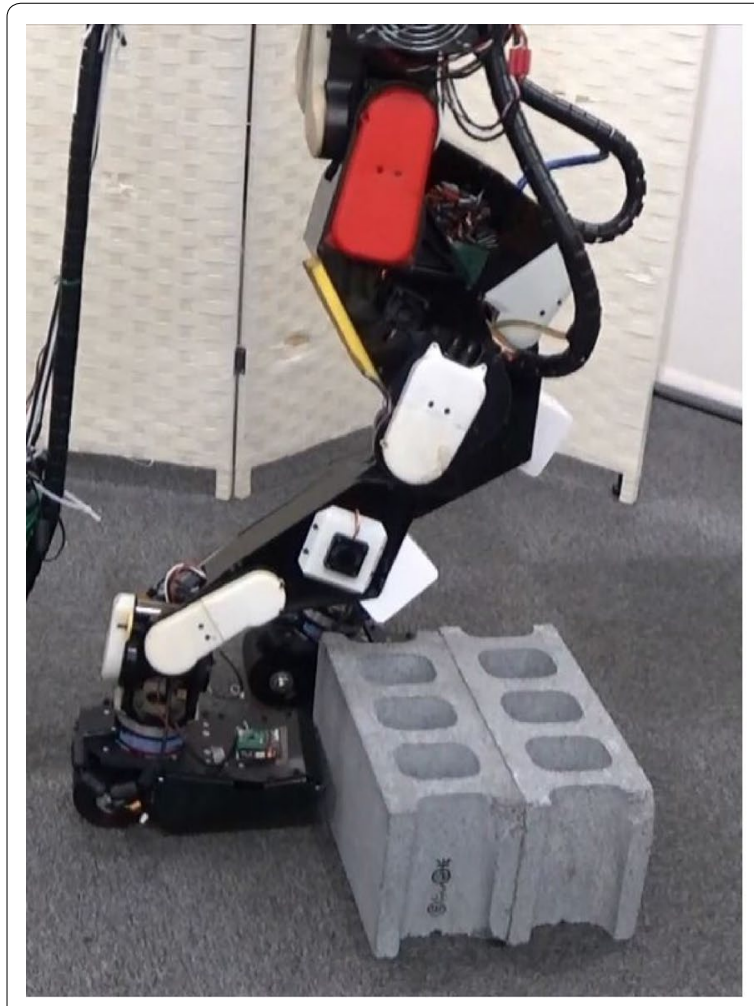

a Experiment

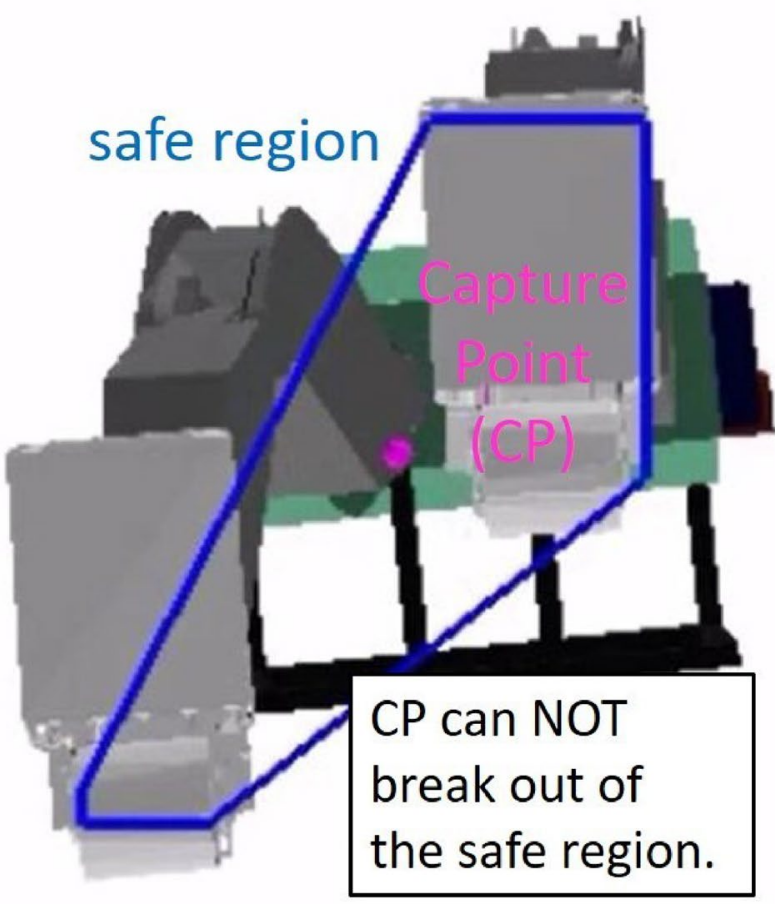

b CP and safe region

Fig. 9 Problem of stumbling 


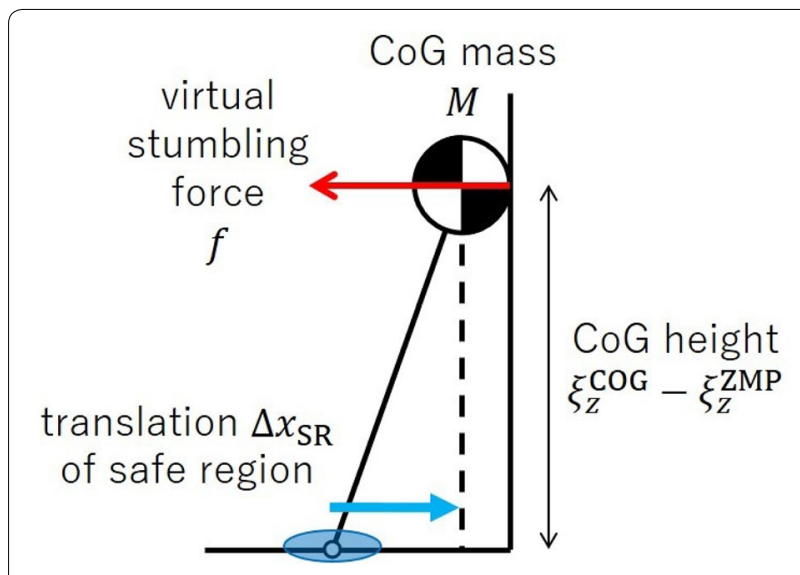

Fig. 10 Force translation of safe region

$M$ of robot. Since the height of center of gravity is represented as $\xi_{z}^{\mathrm{COG}}-\xi_{z}^{\mathrm{ZMP}}, \Delta x_{\mathrm{SR}}$ is defined as follows.

$$
\Delta x_{\mathrm{SR}}=-\frac{\xi_{z}^{\mathrm{COG}}-\xi_{z}^{\mathrm{ZMP}} f}{M g} f
$$

\section{Fall prevention function for leg-wheel transition}

To achieve the locomotion without swing leg, the legwheel transition from two legs to one leg and one wheel is essential. We describe the fall problem in leg-wheel transition and propose two strategies to solve this problem. Figure 11 shows the block diagram of the legwheel transition and the approach to solve the problem that real robot falls in spite of the unchanged target COG (center of gravity). The leg-wheel transition is executed between the stance phase and the phase to which stability margin maximization with lateral constraint $\varepsilon=0$ is applied. The foot sole of robot is rotated around pitch by $20^{\circ}$ with passive wheel on the ground as shown in the upper left of Fig. 11. Note that the robot keeps the height of the passive wheel axle during the leg-wheel transition not to float and press the wheel against the floor. In the stance phase, the target COG is determined in advance from the result of stability margin maximum point $\boldsymbol{p}_{\max }$ calculated in the phase with $\varepsilon=0$. Therefore, the target COG is not changed in the leg-wheel transition between two phases. Even though the target COG in the robot model is not moved, the actual COG of the real robot can be moved and the real robot falls in the real environment. There are several causes of fall such as modeling error as described below. This fall problem of real robot can be solved by the detection of CP that gets out of Safe Region because the velocity of falling affects $\mathrm{CP}$ as shown in Eq. (8). We propose two strategies (1) Online search of lateral constraint and (2) Longitudinal shift of stability margin maximum point to correct the stability margin maximum point based on the detection of $\mathrm{CP}$ so that real robot does not fall.

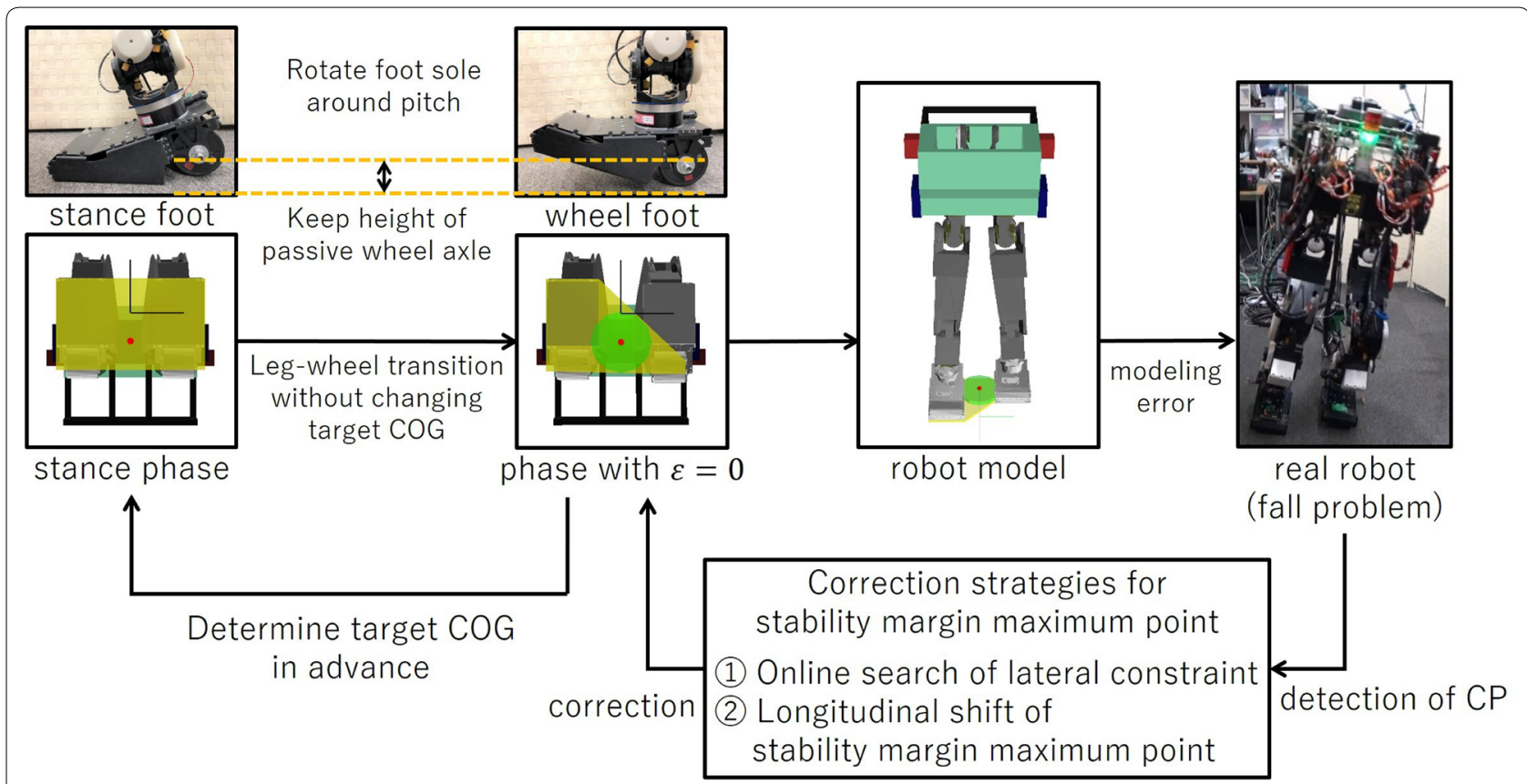

Fig. 11 Block diagram of leg-wheel transition and approach to solve fall problem of real robot 


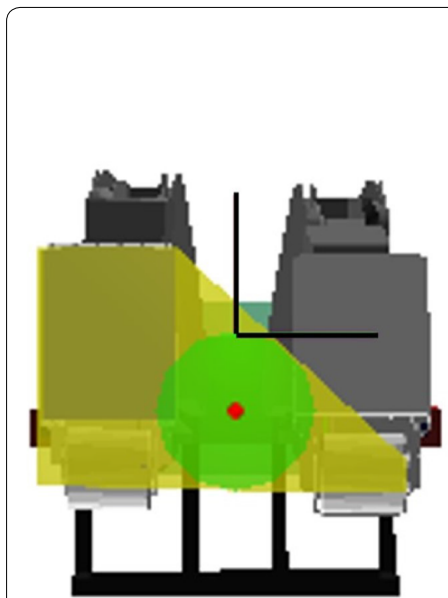

a Phase with $\varepsilon=0$

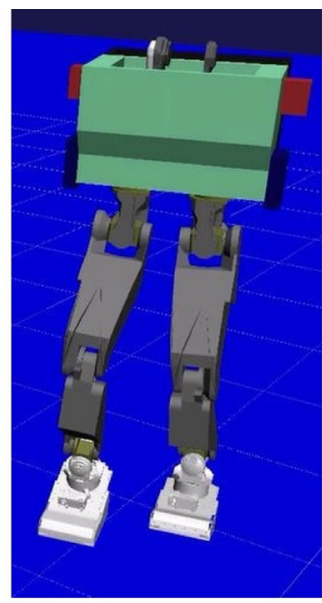

b Simulation

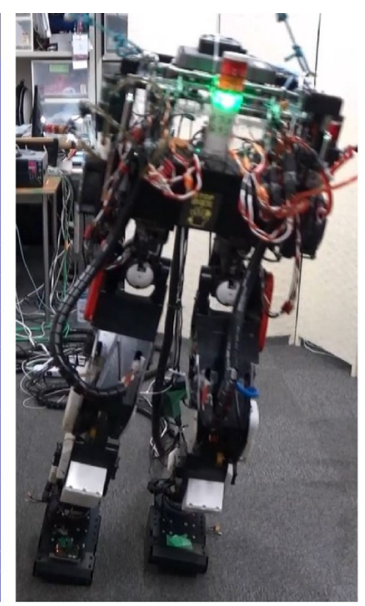

C Experiment

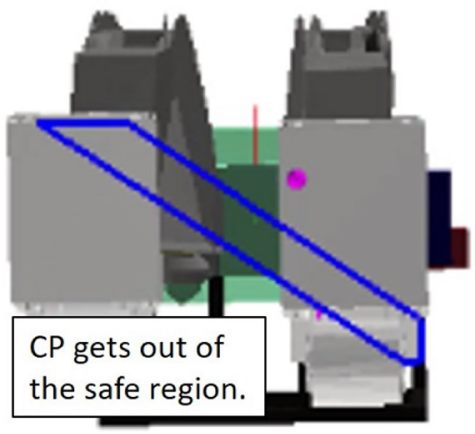

d CP and safe region

Fig. 12 Problem of leg-wheel transition

\section{Problem}

Figure 12a shows the result of applying stability margin maximization with lateral constraint of $\varepsilon=0$. After center of gravity of robot is projected onto the red point in the state that both feet of robot are grounded, the reference joint angles in (A) are commanded to robot. At this time, Fig. 12b shows the dynamics simulation result, Fig. 12c shows the real experimental result, and Fig. 12d shows the state of CP and Safe Region. While the robot succeeds in transition without falling in (B) Simulation, the robot falls in (C) Experiment. From the data (D) in this moment, fall detection is confirmed because CP gets out of the Safe Region. After Emergency Stopper halts the transition motion based on fall detection, the execution of motion reverting [12] enables the fall prevention.

However, because leg-wheel transition is not accomplished by only motion reverting, additional strategies are required. In particular, online-based behavior strategies are desired against the reduction of stability margin $S$ due to the too strong constraint such as $\varepsilon=0$, the deflection of joints of real robot, the error of end effector, and the modeling error regarding contact area of ground and wheel.

\section{Online search of lateral constraint}

This is the online-based technique to search the minimum limit value $\varepsilon$ in lateral constraint Eq. (7) required for achievement of leg-wheel transition. Let $\varepsilon_{0}=0$ be initial value of $\varepsilon$, and $\varepsilon_{k}$, which is $k$-th search result for $\varepsilon$, is obtained sequentially based on the update rule Eq. (10). Note that $k$ is updated to $k+1$ every time CP gets out of Safe Region.

$$
\varepsilon_{k+1}=\varepsilon_{k}+\eta \exp \left\{-\left(T_{k}^{\text {Fall }}-T_{k}^{\text {Start }}\right)\right\}
$$

Let $\eta>0, T_{k}^{\text {Start }}$, and $T_{k}^{\text {Fall }}$ be the learning rate, the start time of leg-wheel transition for $\varepsilon=\varepsilon_{k}$, and the time CP gets out of Safe Region, respectively. $T_{k}^{\text {Fall }}-T_{k}^{\text {Start }}$ is equivalent to the elapsed time (s) from the start of transition to the moment robot is likely to fall. The larger $\varepsilon$ is, the weaker lateral constraint is. Eventually, $\varepsilon$ converges as $T_{k}^{\text {Fall }}-T_{k}^{\text {Start }} \rightarrow \infty$, which denotes the state robot does not fall. As a device to hasten the convergence, the scale factor $\alpha_{k}$ of Safe Region is changed adaptively for the value of $\varepsilon_{k}$ by the following rule.

$$
\alpha_{k}=\alpha_{0}+\left(\alpha_{\max }-\alpha_{0}\right) \frac{\varepsilon_{k}-\varepsilon_{0}}{\varepsilon_{\max }-\varepsilon_{0}}
$$

Let $\alpha_{0}$ and $\alpha_{\max }$ be lower limit and upper limit of $\alpha$, respectively. In this paper, we set $\alpha_{0}=0.6, \alpha_{\max }=1.0$. $\varepsilon_{\max }$ represents the value of $|y|$ of stability margin maximum point without lateral constraint. According to Eq. (11), Safe Region is expanded as search for $\varepsilon_{k}$ proceeds, and $T_{k}^{\text {Fall }}-T_{k}^{\text {Start }}$ also grows larger.

\section{Longitudinal shift of stability margin maximum point}

As opposed to the preceding paragraph, this is the onlinebased technique to achieve leg-wheel transition without changing the constraint $\varepsilon=0$ and without lateral movement. In this technique, without shifting $y$ element of stability margin maximum point $(x, y), x$ element regarding longitudinal direction is shifted by the following shift amount $\Delta x_{\text {shift }}$.

$$
\Delta x_{\text {shift }}=-\left(\left.\xi_{x}^{\mathrm{COG}}\right|_{\text {Fall }}-\left.\xi_{x}^{\mathrm{COG}}\right|_{\text {Start }}\right)
$$


Let $\left.\xi_{x}^{\mathrm{COG}}\right|_{\text {Start }}$ and $\left.\xi_{x}^{\mathrm{COG}}\right|_{\text {Fall }}$ be $x$-coordinate of center of gravity of robot at the time leg-wheel transition starts and the time CP gets out of Safe Region, respectively. Based on the center of gravity error $\left.\xi_{x}^{\mathrm{COG}}\right|_{\text {Fall }}-\left.\xi_{x}^{\mathrm{COG}}\right|_{\text {Start }}$ in real environment, $x$ element of stability margin maximum point is shifted and modified by this offset. The first feature of Eq. (12) is having no lateral movement. The second feature is that robot can achieve leg-wheel transition for the second time after the first trial to obtain $\left.\xi_{x}^{\mathrm{COG}}\right|_{\text {Start }},\left.\xi_{x}^{\mathrm{COG}}\right|_{\text {Fall }}$ because this proposed technique is not iteration-based.

\section{Experiments}

\section{Locomotion experiment based on stability margin} maximization

We show the locomotion experiment by applying stability margin maximization with lateral constraint to a real bipedal robot in Fig. 13. The weight of bipedal robot is $63.9 \mathrm{~kg}$. The feet of robot are equipped with passive wheels without actuators as shown in Fig. 1. The radius of wheel is $44 \mathrm{~mm}$, the width of wheel is $90 \mathrm{~mm}$, and the surface of wheel is made of rubber. The radius of wheel is chosen as small as possible so that the wheel can be stored inside the foot and the robot is safer against slipping, whereas it needs to be chosen not too small so that the contact area between wheel and ground is larger enough to secure support polygon. Because we aim the minimum configuration that wheels can be attached simply to the feet of bipedal robot, the number of passive wheels is defined as one wheel for one foot. And we determine the width of wheel to be the same as the width of foot so that the bipedal robot does not become unstable around roll. The upper part of Fig. 13 shows each phase for locomotion planning of center of gravity projection marked in red point on the bottom view of robot. The lower part of Fig. 13 shows the snapshots of real experiment on the flat carpet floor and the slippery tile floor for the corresponding phases. In the lower part of Fig. 13, timestamps are shown when we set the start time on Phase1 as $0.0 \mathrm{~s}$. In this experiment, we set lateral constraint as $\varepsilon=55$. One cycle of this locomotion is composed of 18 phases and stability margin maximization with lateral constraint is applied on Phase $3,4,7,8,11,12,15,16$. On the phases that both feet of robot are grounded before and after these leg-wheel phases, the transition is executed without changing center of gravity projection point such as $2 \rightarrow 3$ and $4 \rightarrow 5$. The achievement of locomotion based on the transition of leg-wheel phases is observed in the snapshots of real experiment. The passive wheels of real bipedal robot slide $0.36 \mathrm{~m}$ in $1.8 \mathrm{~s}$ for $3 \rightarrow 4$ and $15 \rightarrow 16$, and slide $0.72 \mathrm{~m}$ in $3.6 \mathrm{~s}$ for $7 \rightarrow 8$ and $11 \rightarrow 12$.

To consider the efficiency of locomotion, we compare the electric power and energy consumed by the locomotion based on stability margin maximization with utilizing passive wheel with by the locomotion based on single leg stance as shown in Fig. 6 without utilizing passive wheel. Figure 14 shows the change of electric power consumed by the power supply for robot servo during one cycle locomotion from Phase1 to Phase18. Figure 14a shows the power consumption $P_{\text {Single }}$ for the locomotion by switching the right and left single stance phases without utilizing passive wheel. Figure $14 \mathrm{~b}$ shows the power consumption $P_{\mathrm{SMM}}$ for the locomotion by applying stability margin maximization with lateral constraint of

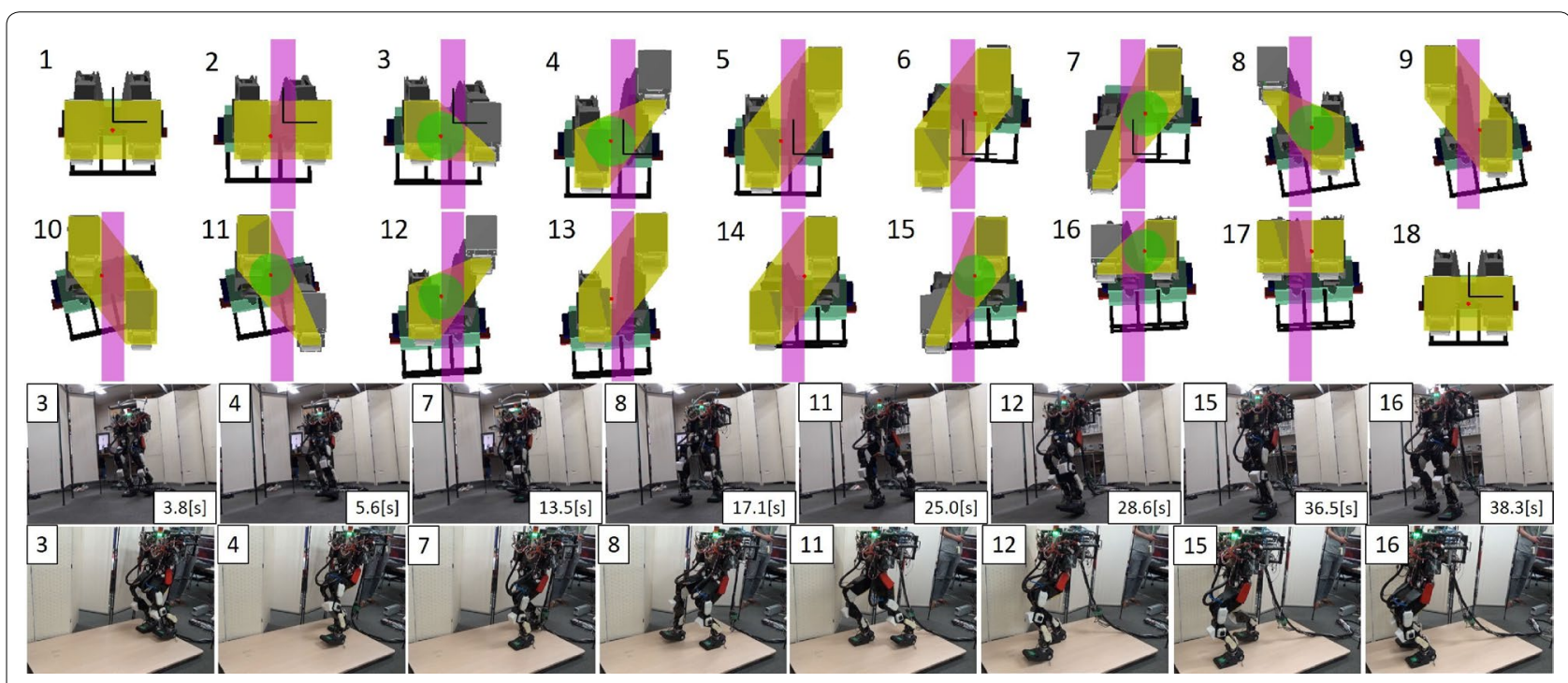

Fig. 13 Locomotion based on stability margin maximization with lateral constraint $\varepsilon=55$ 


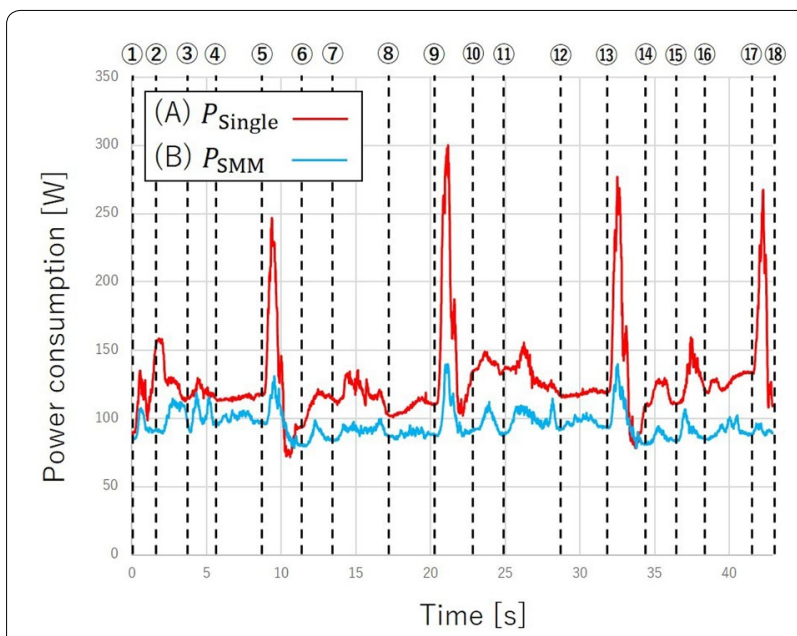

Fig. 14 Power consumption $P_{\{\text {Single,SMm }}$ in one cycle (Phase1Phase18)

$\varepsilon=55$ with utilizing passive wheel. These are compared under the same conditions of the locomotion distance of robot and the transition time among each phase. On Phase5-Phase6, Phase9-Phase10, Phase13-Phase14, and Phase17-Phase18, the unnecessary power consumption is observed in (A) $P_{\text {Single, }}$ which requires the large movement of center of gravity to switch single stance phases. By contrast, in (B) $P_{\text {SMM }}$ based on stability margin maximization, the cooperative use of support regions of foot and wheel contributes to the achievement of efficient locomotion without the unnecessary movement of center of gravity. On Phase3-Phase4, Phase7-Phase8, Phase11Phase12, and Phase15-Phase16 that are the phases to slide wheels, it is observed that $P_{\mathrm{SMM}}$ is smaller than $P_{\text {Single. }}$ It is inferred that this effect reducing power consumption is brought by the weight load distribution to grounded wheel based on stability margin maximization as shown in the result of $F_{z}^{\mathrm{R}}: F_{z}^{\mathrm{L}}$ in Table 1.
To evaluate these locomotion, we compare the energy consumption $E_{\{\text {Single,SMM }}$ during locomotion under the conditions of the same distance and the same time as calculated by the following formula.

$$
E_{\{\text {Single }, \mathrm{SMM}\}}=\int_{0}^{T_{\text {cycle }}} P_{\{\text {Single }, \mathrm{SMM}\}} d t
$$

$T_{\text {cycle }}$ represents the locomotion time for one cycle from Phase1 to Phase18. According to the comparison conditions, we set $T_{\text {cycle }}=43 \mathrm{~s}$ for both $P_{\text {Single }}$ and $P_{\text {SMM }}$. By calculating the value of $E_{\{\text {Single,SMM }}$ in Eq. (13) from the results of power consumption $P_{\{\text {Single,SMM }\}}$ in Fig. 14, we obtain the results of $E_{\text {Single }}=5499.49 \mathrm{~J}$ and $E_{\mathrm{SMM}}=4093.29 \mathrm{~J}$. These results denote $E_{\text {Single }}>E_{\mathrm{SMM}}$ and stability margin maximization succeeds in reducing energy consumption during locomotion by approximately $1400 \mathrm{~J}$.

Leg-wheel robots have the road ability of legged robots to travel over various environments and the stable and efficient mobility of wheeled robots. The leg-wheel robot to utilize passive wheels based on the proposed method can achieve these ability and mobility by locomotion with pushing obstacles in the environment where disturbance is mixed. To clarify whether this leg-wheel robots rather than general bipedal robots are useful for specific task, we compare (A) General bipedal walking with (B) Proposed locomotion approach for the locomotion tasks with pushing obstacles out in the debris environment as shown in Fig. 15. Figure 15 shows the dynamics simulation results that the bipedal robot and the leg-wheel robot try to pass through the debris environment where $5 \mathrm{~kg}$ of cuboid obstacle, $5 \mathrm{~kg}$ of cylinder obstacle, and $3 \mathrm{~kg}$ of cuboid obstacle are mixed. In Fig.15a, the robot starts bipedal walking with swing leg and tries to push the obstacles by walking. However, the robot loses balance while pushing three heavy obstacles by swing leg in Fig. 15a-4 and the robot falls in Fig. 15a-5 and fails in pushing

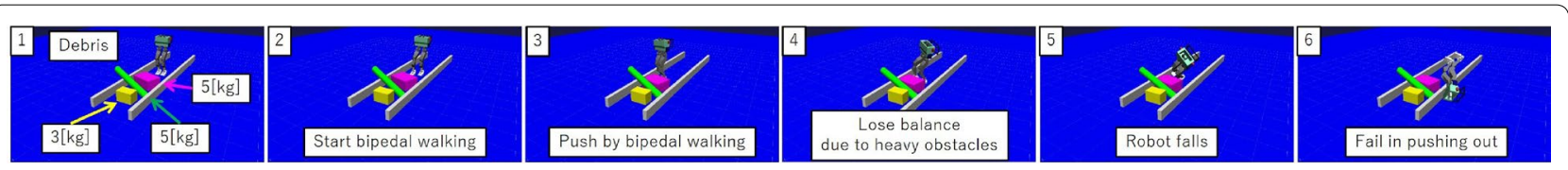

a General bipedal walking
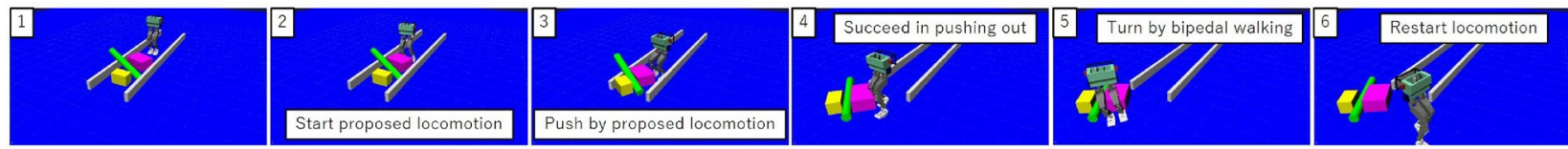

b Proposed locomotion approach

Fig. 15 Locomotion tasks with pushing obstacles out in debris environment 
the debris obstacles out in Fig. 15a-6. By contrast, in Fig. 15b, the robot starts proposed locomotion utilizing passive wheel without swing leg and succeeds in pushing the debris obstacles out. The leg-wheel robot is able to turn by bipedal walking in Fig. $15 \mathrm{~b}-5$ and restart this locomotion toward the different direction in Fig. 15b-6. These comparison results demonstrate the robust locomotion skill in a debris environment for the proposed locomotion approach utilizing passive wheel. The locomotion ability of this leg-wheel robot to push obstacles away is demonstrated in real environment. In Fig. 16, $3.4 \mathrm{~kg}, 3.8 \mathrm{~kg}, 5.5 \mathrm{~kg}$, and $8.3 \mathrm{~kg}$ of the mixed obstacles are placed randomly on the road. It is observed that the real leg-wheel robot moves while pushing obstacles away. Stability margin maximization contributes to the response to disturbance during locomotion and the improvement of robustness.

\section{Fall prevention experiment for stumbling}

Although the robustness is secured by stability margin maximization, robot stumbles in the case that the larger disturbance exists. We show the fall prevention experiment for this case in Fig. 17. In Fig. 17-1, $13.9 \mathrm{~kg}$ * 2 pieces of the concrete blocks are placed side by side on the road and the robot can not push these obstacles away due to the heavy weight. For this situation, Fig. 17-2 shows the state that force translation of Safe Region works. In Fig. 17-2, the Safe Region is translated forward by $\Delta x_{\mathrm{SR}}=243.2 \mathrm{~mm}$ and Emergency Stopper works since CP gets out of the Safe Region. After Emergency Stopper works, the robot slides the passive wheel of stumbling foot backward and the emergency stopping to prevent fall is achieved so that CP gets inside the Safe Region as shown in Fig. 17-3.

\section{Online search experiment of lateral constraint}

We set the initial value of lateral constraint as $\varepsilon_{0}=0$ and show the result of online search of the value $\varepsilon$ so that the real robot can achieve leg-wheel transition on the carpet floor without falling in Fig. 18. Figure 18 shows the results of stability margin maximization for $\varepsilon=\varepsilon_{k}$ and the moments CP gets out of the Safe Region, where CP is inside the Safe Region when the online search is converged. The elapsed time $\Delta T_{k}=T_{k}^{\text {Fall }}-T_{k}^{\text {Start }}$ from the start of leg-wheel transition to these moments is also shown in Fig. 18. Note that the leg-wheel transition is expected to be executed as Phase $2 \rightarrow 3$ in Fig. 13 for 5 s. Regarding the way to determine $\eta$ in the update rule Eq. (10), we can set $\eta=138.542$ from the relationship of Eq. (10) so that $\varepsilon_{k+1}-\varepsilon_{k}$, which is the change of $\varepsilon$, equals 1 $\%$ of the maximum range $\varepsilon_{\max }-\varepsilon_{0}=93.3491$ when the sufficient time $\Delta T_{k}=5 \mathrm{~s}$ for transition elapses. From the search experiment under the above conditions, we obtain the result that $\varepsilon$ eventually converges with $\varepsilon_{5}=54.0728$ after 6 trials. This convergence value is close to the setting value $\varepsilon=55$ applied in Fig. 13 and the real bipedal robot is able to achieve locomotion as a result.

We also show the experiment of fall prevention for leg-wheel transition and then moving on the slippery tile floor in Fig. 19. In Fig. 19(1)-(4), the online search of lateral constraint $\varepsilon$ is executed on the tile floor and $\varepsilon$ converges with $\varepsilon_{3}=23.095$ after only 4 trials. In Fig.19(5)-(8), stability margin maximization with the obtained lateral constraint $\varepsilon=23.095$ enables the robot to succeed in moving so that CP does not get out of the Safe Region. It is inferred that the smaller convergence value of $\varepsilon$ and fewer trials than the previous experimental result shown in Fig. 18 are caused by the floor conditions (the difference between elastic carpet floor and rigid tile floor). The leg-wheel transition is achieved without

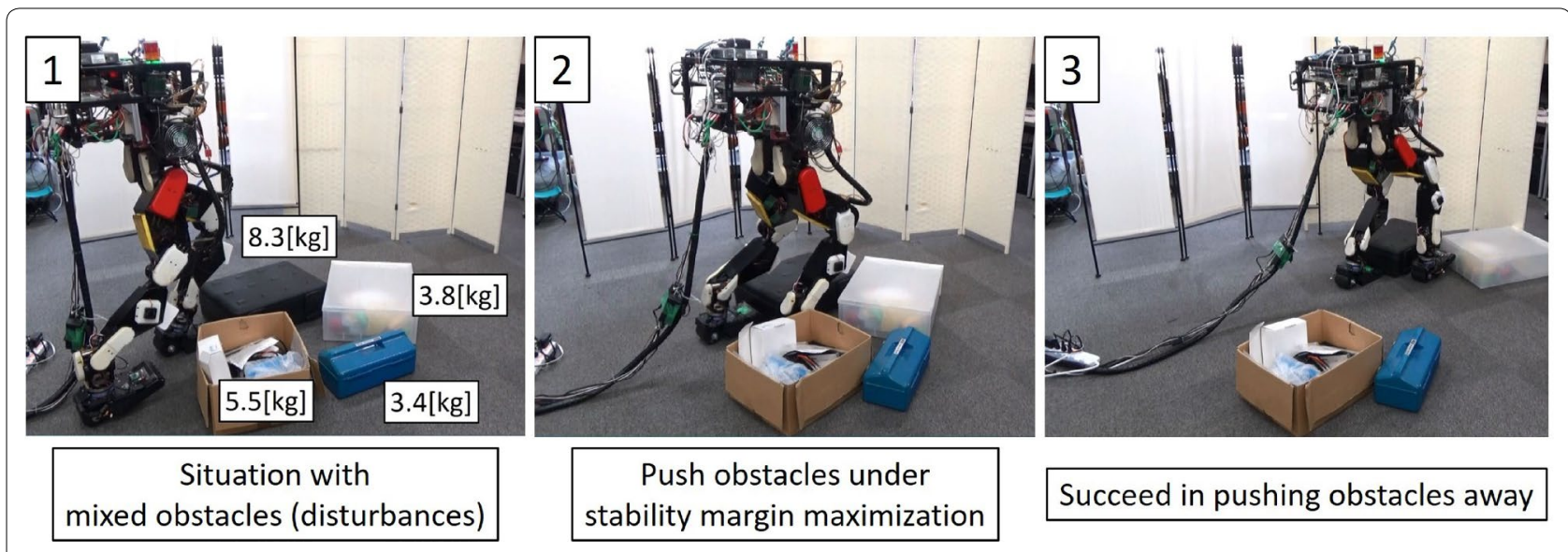

Fig. 16 Locomotion experiment with pushing obstacles away 


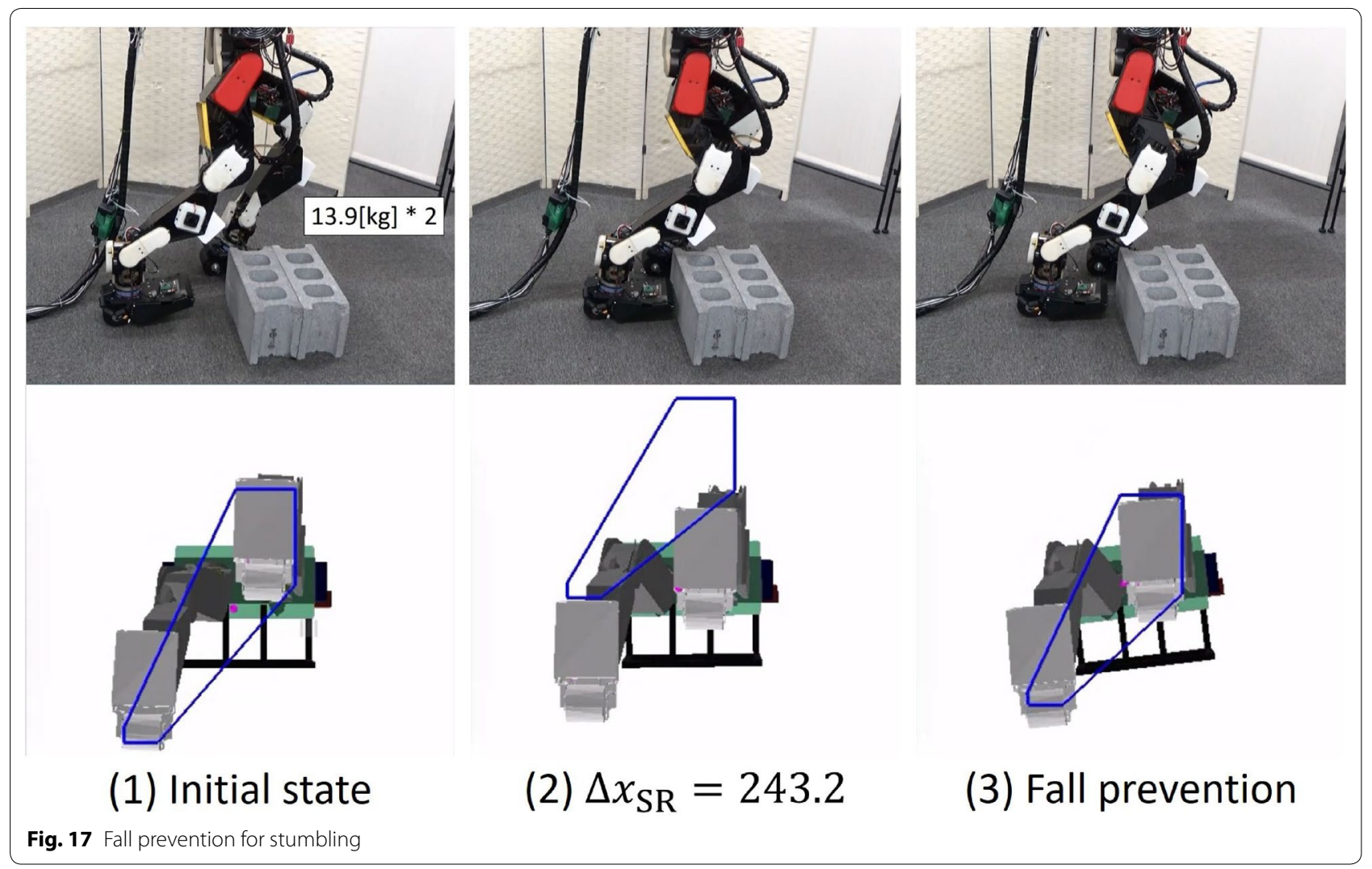

slipping and falling and the leg-wheel robot can move robustly regardless of the difference of floor surface.

\section{Longitudinal shift experiment of stability margin maximum point}

In order to achieve leg-wheel transition without falling, besides the approach to change lateral constraint, it is effective to shift the stability margin maximum point which center of gravity of robot is projected onto in the longitudinal direction as shown in Fig. 20. Figure 20 shows the plot regarding the change of longitudinal position $\xi_{x}^{\mathrm{COG}}$ of center of gravity. At time $0 \mathrm{~s}$, the legwheel transition with $\varepsilon=0$ starts. At time $T_{1}=2.24$ $\mathrm{s}$, Emergency Stop works since CP gets out of the Safe Region. From time $T_{1}=2.24 \mathrm{~s}$ to time $T_{2}=6.08 \mathrm{~s}$, the motion reverting to previous phase is executed. At time $T_{3}=12.79 \mathrm{~s}$, the longitudinal shift of stability margin maximum point is achieved so that $\mathrm{CP}$ gets inside the Safe Region. The longitudinal shift amount $\Delta x_{\text {shift }}=-42.47 \mathrm{~mm}$ is obtained during the period from time $0 \mathrm{~s}$ to time $T_{1}=2.24 \mathrm{~s}$ based on Eq. (12). After the stability margin maximum point is shifted by $\Delta x_{\text {shift }}$ at time $T_{2}=6.08 \mathrm{~s}$ when motion is reverted to previous phase, the leg-wheel transition with $\varepsilon=0$ is executed without falling during the period from time $T_{2}=6.08 \mathrm{~s}$ to time $T_{3}=12.79 \mathrm{~s}$.

Locomotion with $\varepsilon=0$ is also achieved by utilizing the obtained longitudinal shift amount $\Delta x_{\text {shift }}$. Fig. 21 shows the plots regarding the change of lateral position of center of gravity during the locomotion applied by stability margin maximization without lateral constraint ("no constraint"), stability margin maximization with lateral constraint of $\varepsilon=55$ ("with $\varepsilon=55$ "), and longitudinal shift of stability margin maximum point with $\varepsilon=0$ ("with $\varepsilon=0$ "), respectively. It is observed that the effectiveness for prevention of body swing sideways is heightened in this order. Utilizing the locomotion with $\varepsilon=0$, the real bipedal robot is able to achieve the passing through narrow space as shown in Fig. 22. In Fig. 22a applied by stability margin maximization with lateral constraint of $\varepsilon=55$, the interference between the real robot and the wall of narrow space is observed. By contrast, in Fig. 22b during the locomotion with $\varepsilon=0$, minimizing the lateral amplitude enables the real robot to pass through narrow space without the collision with walls. 


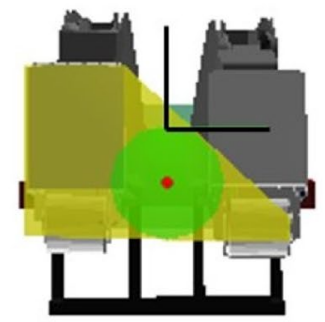

$$
\varepsilon_{0}=0
$$

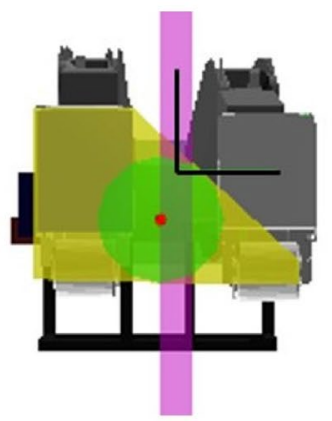

$$
\varepsilon_{2}=23.7517
$$

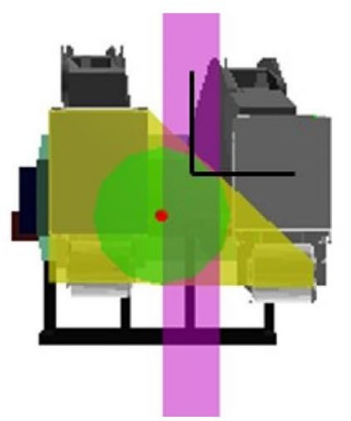

$$
\varepsilon_{4}=44.6696
$$
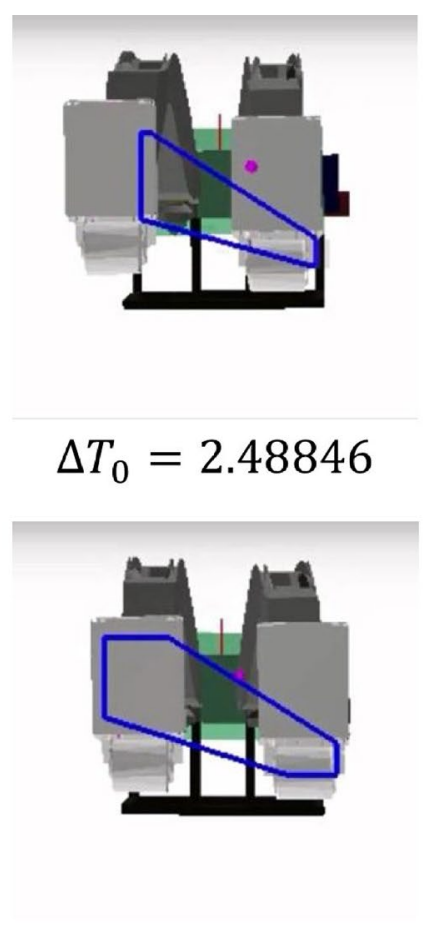

$\Delta T_{2}=2.47956$

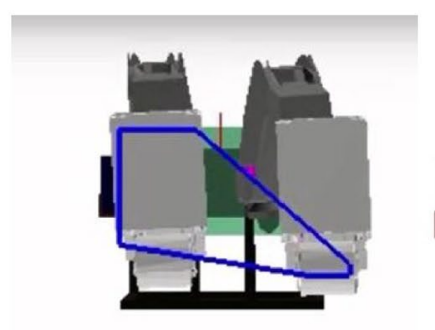

$\Delta T_{4}=2.69012$

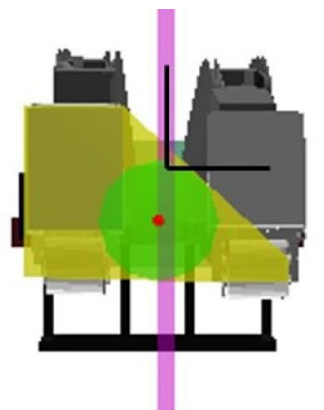

$$
\varepsilon_{1}=11.5042
$$
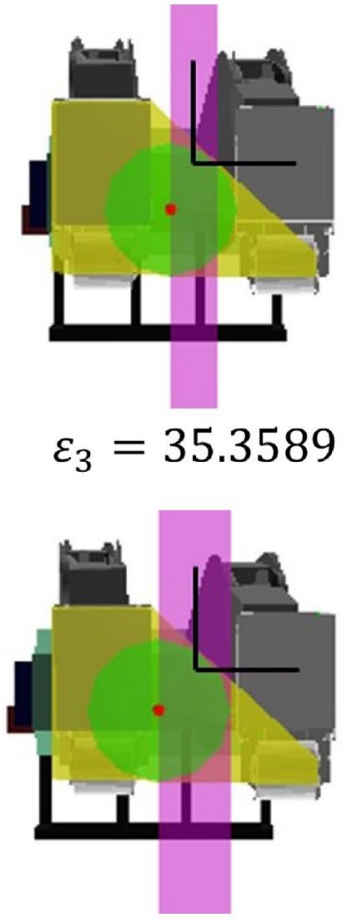

$$
\varepsilon_{5}=54.0728
$$

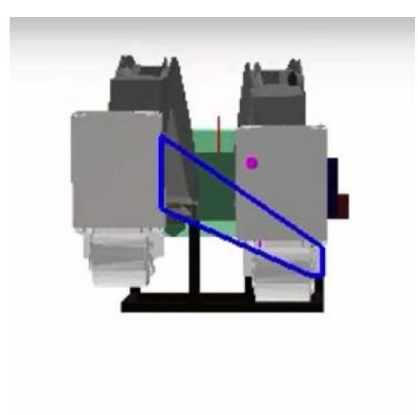

$\Delta T_{1}=2.42585$

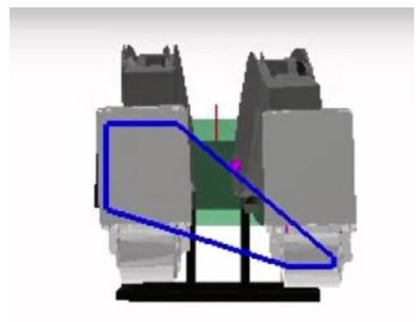

$\Delta T_{3}=2.70001$

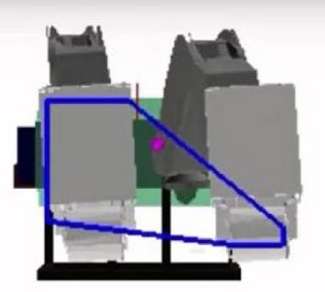

Converged

Fig. 18 Online search of lateral constraint $\varepsilon$

\section{Conclusion}

This paper established the locomotion approach without swing leg by utilizing passive wheels attached to the feet of bipedal robot without wheel actuators. The proposed locomotion approach provided the effectiveness of the stability based on expansion of support polygon during locomotion, the robustness for external obstacles and emergency stopping not to fall, and the adaptability to prevent body swing sideways. To achieve these features, this paper proposed stability margin maximization and fall prevention functions. Stability margin maximization is the optimization approach how to project the center of gravity of robot onto the support polygon formed from sole and wheel. Fall prevention functions are the strategies against the fall factors of real robot in the real environment that is a difficult situation to prevent fall by the only offline planning. Finally, this paper demonstrated the experiments using real bipedal robot to verify these effects. In particular, the experiments in this paper proved the remarkable outcome that stability margin maximization in the proposed approach can reduce power and energy consumption during locomotion. This lower power and energy consumption for the proposed locomotion is revealed from the experimental results as shown in Fig. 14 and it contributes to the improvement of locomotion efficiency of bipedal robot. 


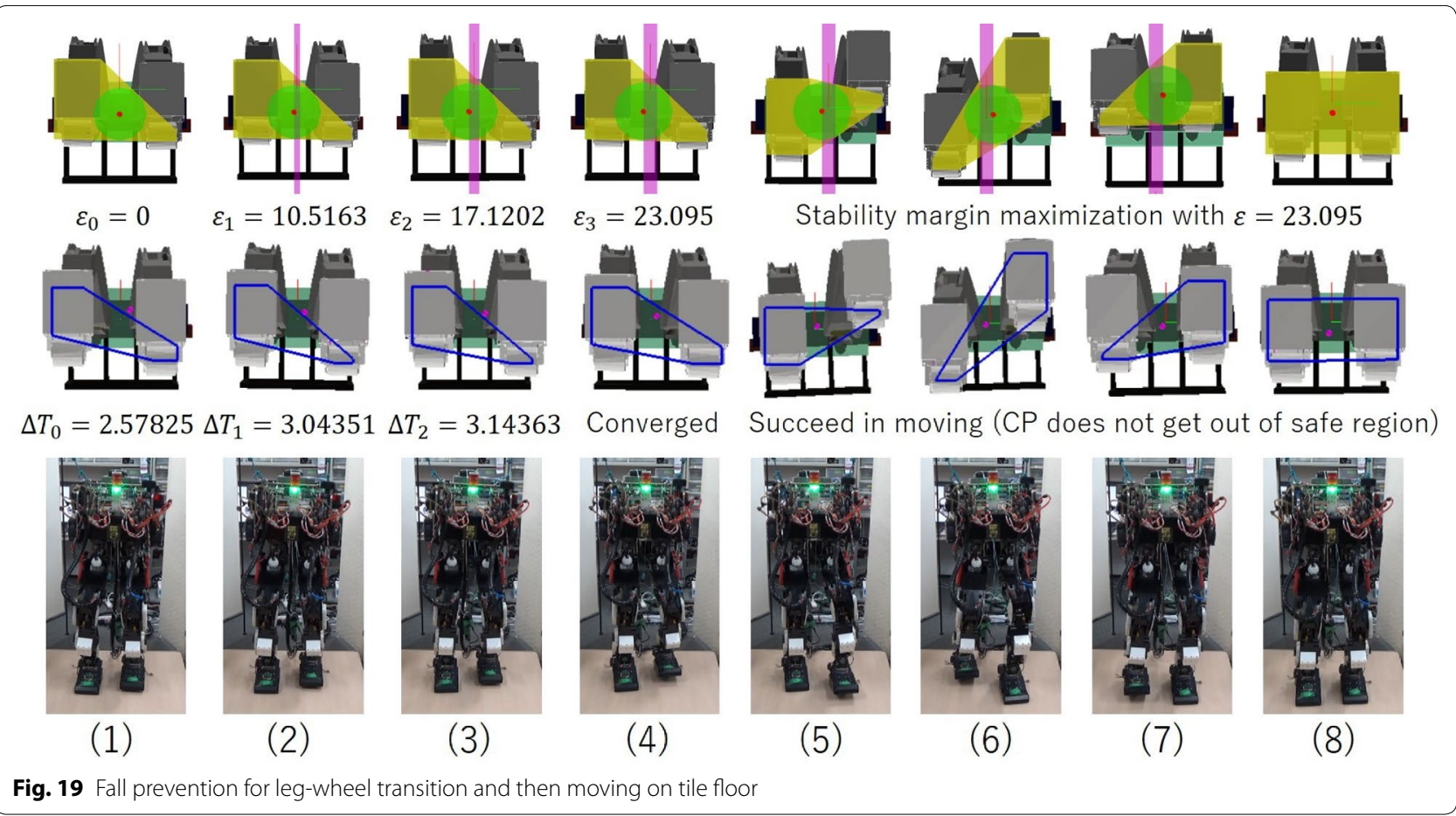

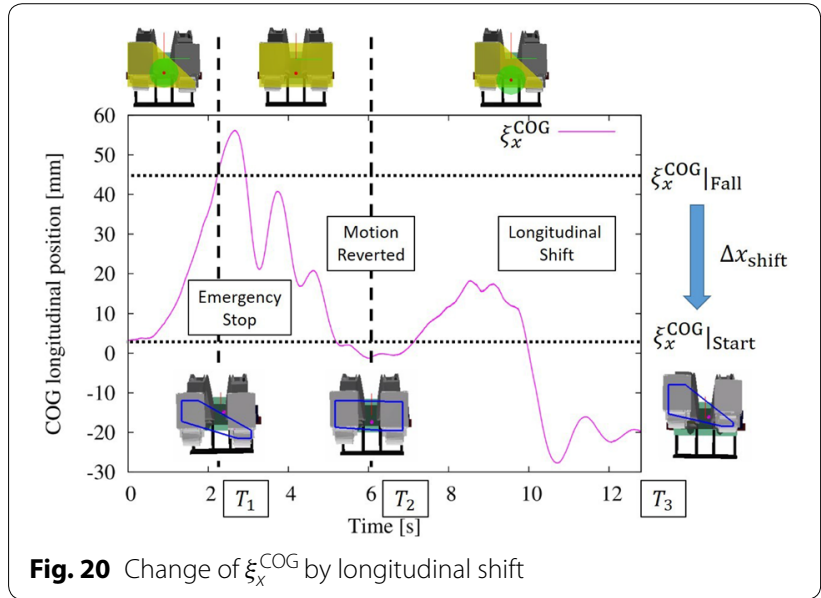

This paper expanded locomotion style of leg-wheel robots without introducing complex mechanisms to legged robots, adding actuators, and modifying configuration of electrical system. On the other hand, there are some disadvantages as trade-offs such as (1) slow locomotion, (2) risk of backward falling due to passive wheels on the heel of robot, and (3) limits of robustness for floor conditions. Regarding (1), improvement of locomotion speed is expected and it is required to expand from static locomotion strategies to dynamic locomotion strategies. This can be feasible by replacing center of gravity trajectories with

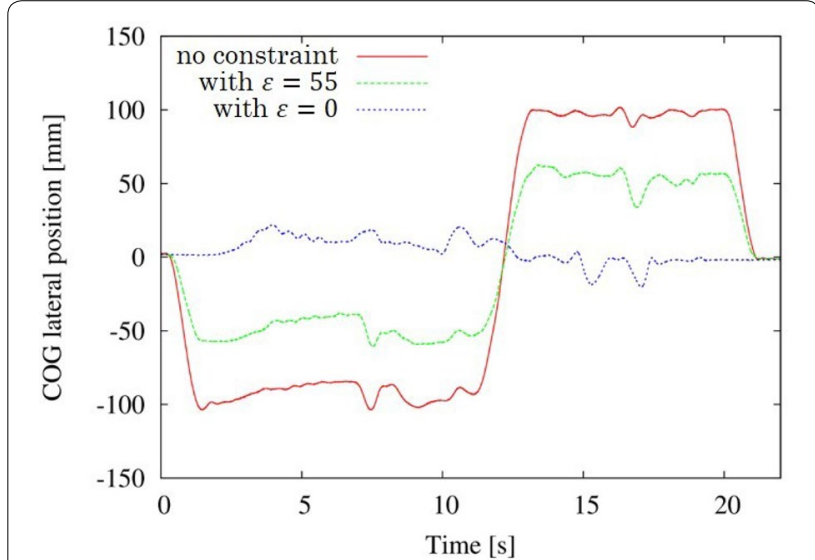

Fig. 21 Comparison of COG lateral change

ZMP trajectories, which are planned by stability margin maximization proposed in this paper. Regarding (2), this paper showed the evidence that robot does not fall backward even when center of gravity of robot locates posterior to edge of feet soles in Fig. 4. However, the more terrible modeling error between robot model and real robot and the larger disturbance can affect the risk of backward falling. We consider that the switching between active wheels and passive wheels is required to brake robot and prevent falling against these external factors. Regarding (3), this paper demonstrated the locomotion experiment in Fig. 13 and the 


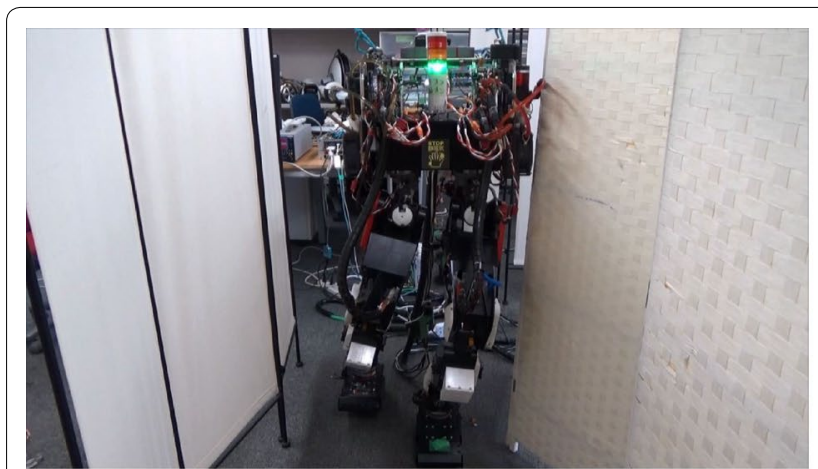

a Failed with $\varepsilon=55$

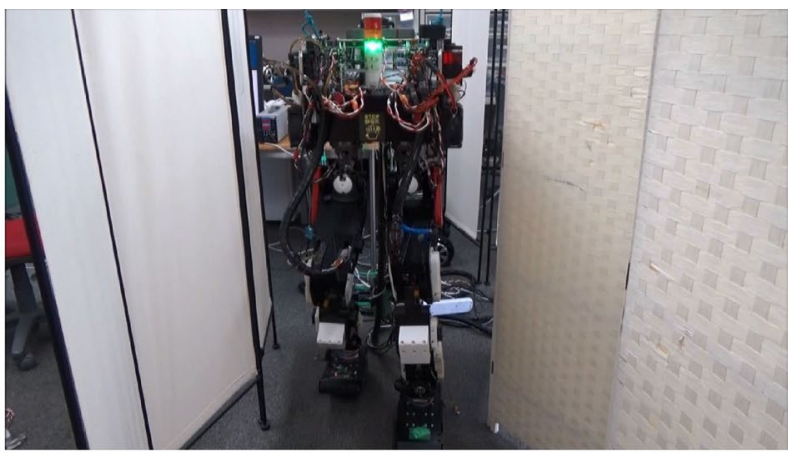

b Succeeded with $\varepsilon=0$

Fig. 22 Passing through narrow space

fall prevention for leg-wheel transition in Fig. 19 under the different floor conditions (on the rough carpet floor and on the slippery tile floor). The difference in these floor conditions is flat floor surface and further consideration is needed in order to validate the proposed locomotion approach on sloping terrain and confirm the limits of robustness of this leg-wheel robot.

\section{Acknowledgements}

This work was partially supported by the JSPS KAKENHI Grant Number 19 K20373 and the Industry-Academia Collaborative Research of The University of Tokyo and Panasonic Corporation.

\section{Authors' contributions}

KK conceived the locomotion approach, implemented the strategies, carried out the experiments, and wrote this manuscript as corresponding author. $\mathrm{NI}$ supervised this research as cooperative research group. SN supported the idea, the programming, and the experiments. $\mathrm{YK}, \mathrm{KO}$, and $\mathrm{Ml}$ supervised this research and gave advice for this study, the idea, and the experiments. All authors read and approved the final manuscript.

\section{Funding}

This work has been supported by the Japan Society for the Promotion of Science (19K20373) and the Industry-Academia Collaboration in The University of Tokyo.

\section{Availability of data and materials}

The datasets during and/or analysed during the current study available from the corresponding author on reasonable request.

\section{Ethics approval and consent to participate}

Not applicable.

\section{Consent for publication}

Not applicable.

\section{Competing interests}

The authors declare that they have no competing interests.

\section{Author details}

${ }^{1}$ Department of Mechano-Informatics, Graduate School of Information Science and Technology, The University of Tokyo, 7-3-1, Hongo, Bunkyo-ku, Tokyo 113-8656, Japan. ${ }^{2}$ Robotics Promotion Office, Manufacturing Innovation Division, Panasonic Corporation, 8-21-1, Ginza, Chuo-ku, Tokyo 104-0061, Japan.
Received: 30 March 2020 Accepted: 3 October 2020

Published online: 15 October 2020

\section{References}

1. Endo G, Hirose S (2000) Study on Roller-Walker (multi-mode steering control and self-contained locomotion). In: Proceedings of the 2000 IEEE International Conference on Robotics and Automation, pp 2808-2814

2. Hashimoto K, Hosobata T, Sugahara Y, Mikuriya Y, Sunazuka H, Kawase M, Hun-ok Lim, Takanishi A (2005) Realization by biped leg-wheeled robot of biped walking and wheel-driven locomotion. In: Proceedings of the 2005 IEEE International Conference on Robotics and Automation, pp 2970-2975

3. Lim J, Lee I, Shim I, Jung H, Joe HM, Bae H, Sim O, Oh J, Jung T, Shin S, Joo K, Kim M, Lee K, Bok Y, Choi DG, Cho B, Kim S, Heo J, Kim I, Lee J, Kwon IS, Oh JH (2017) Robot system of DRC-HUBO+ and control strategy of team KAIST in DARPA Robotics Challenge Finals. J Field Robot 34(4):802-829

4. Vukobratović M, Stepanenko J (1972) On the stability of anthropomorphic systems. Math Biosci 15(1):1-37

5. Pratt J, Carff J, Drakunov S, Goswami A (2006) Capture point: A step toward humanoid push recovery. In: Proceedings of the 2006 6th IEEERAS International Conference on Humanoid Robots, pp 200-207

6. Shirai T, Nagamatsu Y, Suzuki H, Nozawa S, Okada K, Inaba M (2018) Design and evaluation of torque based bipedal walking control system that prevent fall over by impulsive disturbance. In: Proceedings of the 2018 IEEE/RSJ International Conference on Intelligent Robots and Systems, pp 739-746

7. Kojima K, Ishiguro Y, Sugai F, Nozawa S, Kakiuchi Y, Okada K, Inaba M (2017) Rotational sliding motion generation for humanoid robot by force distribution in each contact face. IEEE Robot and Autom Lett 2(4):2088-2095

8. McGhee RB, Frank AA (1968) On the stability properties of quadruped creeping gaits. Math Biosci 3:331-351

9. qPOASES User's Manual Version 3.0 (December 2014). https://www.coinor.org/qpOASES/doc/3.0/manual.pdf

10. Choreonoid Official Site. https://choreonoid.org/en/

11. Flash T, Hogan N (1985) The coordination of arm movements: an experimentally confirmed mathematical model. J Neurosci 5(7):1688-1703

12. Nozawa S, Kuroiwa E, Kojima K, Ueda R, Murooka M, Noda S, Kumagai I, Ohara Y, Kakiuchi Y, Okada K, Inaba M (2015) Multi-layered real-time controllers for humanoid's manipulation and locomotion tasks with emergency stop. In: Proceedings of the 2015 IEEE-RAS 15th International Conference on Humanoid Robots, pp 381-388

13. Xinjilefu X, Feng S, Atkeson CG (2015) Center of mass estimator for humanoids and its application in modelling error compensation, fall 
detection and prevention. In: Proceedings of the 2015 IEEE-RAS 15th International Conference on Humanoid Robots, pp 67-73

14. Atkeson CG, Babu BPW, Banerjee N, Berenson D, Bove CP, Cui X, DeDonato M, Du R, Feng S, Franklin P, Gennert M, Graff JP, He P, Jaeger A, Kim J, Knoedler K, Li L, Liu C, Long X, Padir T, Polido F, Tighe GG, Xinjilefu X (2015) No falls, no resets: Reliable humanoid behavior in the DARPA robotics challenge. In: Proceedings of the 2015 IEEE-RAS 15th International Conference on Humanoid Robots, pp 623-630

\section{Publisher's Note}

Springer Nature remains neutral with regard to jurisdictional claims in published maps and institutional affiliations.

\section{Submit your manuscript to a SpringerOpen ${ }^{\circ}$ journal and benefit from:}

- Convenient online submission

- Rigorous peer review

- Open access: articles freely available online

- High visibility within the field

- Retaining the copyright to your article

Submit your next manuscript at $\boldsymbol{\nabla}$ springeropen.com 